\title{
Trabajando en Proyectos con Lego Serious Play ${ }^{\circledR}$
}

\author{
Guillermo Montero Fernández-Vivancos, Alberto Cerezo Narváez, Manuel Otero Mateo
}

Recibido: 15 de Febrero de 2019 / Aceptado: 09 de Abril de 2019

\section{Resumen}

Esta investigación estudia la formación en dirección de proyectos a través de la metodología Lego Serious Play®, explorando su uso y aspectos complementarios junto a otras técnicas de pensamiento creativo, demostrando su utilidad como complemento de los métodos tradicionales de enseñanza, reforzando conceptos y trabajando en competencias. Para ello, se examinan dos casos de aplicación práctica, analizando los resultados obtenidos, con sus circunstancias específicas. Además, se valora la motivación y experiencia de los participantes, así como la contribución del juego al aprendizaje, evaluando la calidad del proceso y cómo este ayuda a conseguir los objetivos educativos planteados, asegurando su adecuación.

\section{Palabras clave}

gamificación, juegos serios, Lego Serious Play, dirección de proyectos, competencias.

\section{Introducción}

En 1996, la compañía danesa de juguetes LegoTM solicita, a raíz de la crisis en la venta de juegos de construcción, debido al auge de la incipiente y disruptiva industria del videojuego (Kristiansen et al., 2009; Rasmussen, 2010), y como prioridad estratégica, el diseño de un programa que ayude a las reuniones de trabajo entre ejecutivos y directivos a generar ideas para poder revertir esta tendencia. De esta manera, de modo natural, se van incorporando a las reuniones aspectos de juego, construccionismo y constructivismo, y animando a los participantes en el programa a utilizar material de la propia empresa, con el objeto de expresar significados construyendo (Roos et al., 2004). El trabajo colaborativo, junto a la Business School de Lausanne y la Università della Svizzera Italiana de Lugano, ambas en Suiza, lleva a LegoTM a desarrollar un conjunto de principios básicos para emprender proyectos exitosos que caracterizan su compromiso con el

Guillermo Montero Fernández-Vivancos * gmontero@us.es

\footnotetext{
Alberto Cerezo Narváez **

alberto.cerezo@uca.es

Manuel Otero Mateo **

manuel.otero@uca.es

* Universidad de Sevilla, Departamento de Organización

Industrial y Gestión de Empresas II, Escuela Técnica Superi-

or de Ingeniería, Avenida de los Descubrimientos s/n, 41092

Sevilla

** Universidad de Cádiz, Departamento de Ingeniería

Mecánica y Diseño Industrial, Escuela Técnica Superior de

Ingeniería, Avenida de la Universidad de Cádiz, 10, 11519

Puerto Real, Cádiz
}

programa Building value with Lego (2012) que acaba consolidándose como la metodología Lego Serious Play® (en adelante LSP), y que tiene carácter abierto desde 2010 bajo licencia Creative Commons (Lego, 2010):

1. Esclarecimiento de las reglas y expectativas a los grupos de trabajo.

2. Establecimiento de garantías de beneficio mutuo.

3. Distinción estricta entre personal y usuarios.

4. Desarrollo de herramientas de comunicación y foros de interacción.

5. Transparencia.

Desde sus orígenes, la metodología LSP ha sido utilizada, para una amplia variedad de propósitos, incluyendo, entre otros, análisis de la cadena de valor, competitividad, diseño y desarrollo de productos, estrategia organizacional, fusiones y adquisiciones, gestión del cambio, liderazgo y dirección de equipos, segmentación de mercados, etc (Kristiansen et al., 2009). En un contexto académico, las principales áreas formativas en las que se puede aplicar el método son, como resalta el programa europeo Lifelong Learning Program, estrategia, gestión de proyectos, innovación, marketing, recursos humanos, social media, y ventas, entre otros (Mccusker, 2014; Montero, 2018).

El objeto de este artículo, como propone la Figura 1, es desarrollar el concepto de competencias en dirección de proyectos, estimular la adquisición de habilidades para el fomento de un aprendizaje autónomo y entrenar la búsqueda 
de soluciones a los problemas mediante dinámicas de trabajo colaborativo, a través de dos casos de estudio en los que se emprenden una serie de talleres prácticos en los que, por medio de estrategias y técnicas de gamificación, se trabaja la Iniciación de un Proyecto (desarrollada por la Universidad de Sevilla en el Caso 1) y la Gestión de Requisitos (desarrollada por la Universidad de Cádiz en el Caso 2). A nivel estructural, y una vez introducido el tema, se resalta la im- portancia y utilidad que, para los futuros trabajadores, van adquiriendo las competencias en dirección de proyectos. A continuación, se eligen las metodologías seleccionadas para su entrenamiento. Finalmente, se discuten los resultados y exponen las principales conclusiones obtenidas, así como se plantean líneas futuras que den continuidad a la investigación.

Figura 1 Objetivos de la investigación

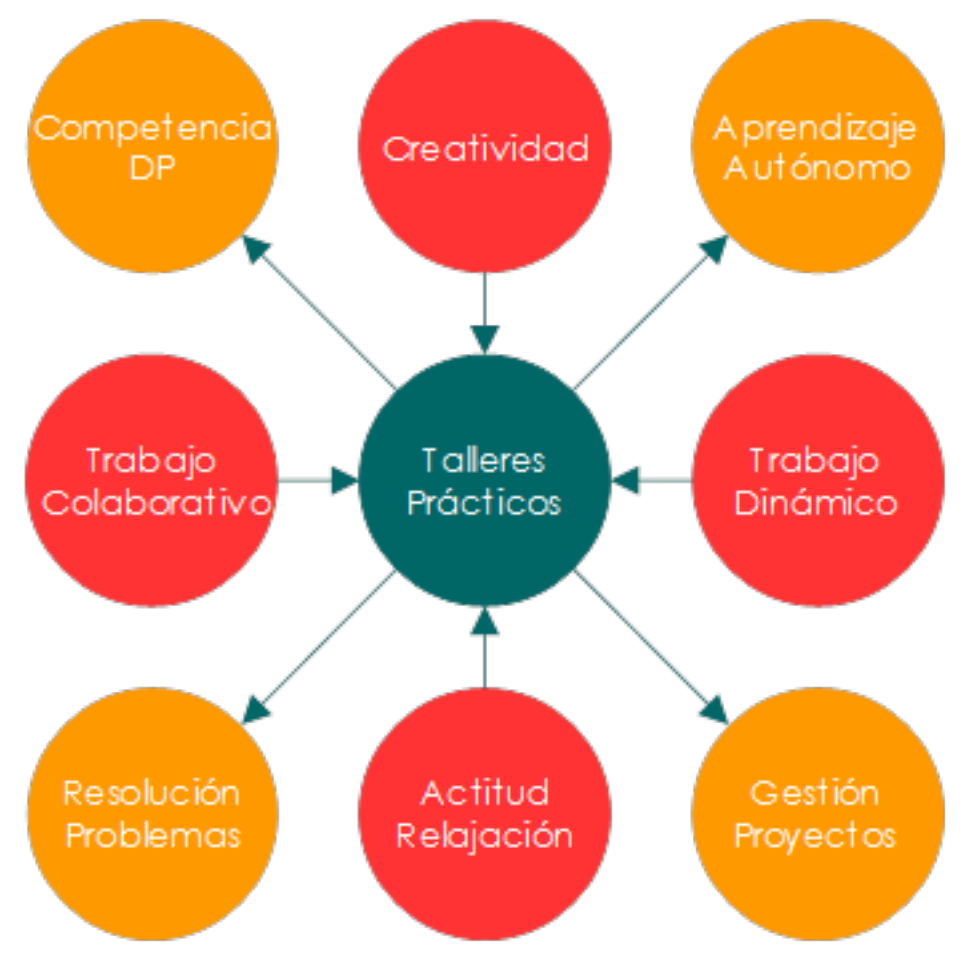

\section{Competencias en dirección de proyec- tos}

Los proyectos se han hecho omnipresentes no solo en la economía sino también en la sociedad (Jensen et al., 2016). Además, en los entornos actuales de trabajo organizacional, el enfoque clásico de carrera ha sido ampliamente reemplazado por nuevos paradigmas que minimizan los factores organizacionales y enfatizan la importancia de ir cultivando un conjunto individual de habilidades (Neubert et al., 2015). En este contexto, la flexibilidad de los modelos de competencias permite adaptar fácilmente posiciones complejas y cambiantes así como abordar las tareas no rutinarias e interactivas que se requieren en estas (Rodríguez et al., 2018). La formación basada en competencias tiene múltiples aplicaciones para el desarrollo de las personas, para las organizaciones y para la propia sociedad en su conjunto, destacando el vínculo entre educación y trabajo (Bonilla, 2008). Por ello, los programas de educación deberían tener como objetivo preparar a los estudiantes para el trabajo (Rainsbury et al., 2002), mediante el desarrollo de competencias genéricas y específicas útiles, tanto para los estudiantes como para los empleadores.
En el ámbito de la dirección de proyectos, la aplicación de conocimientos, herramientas y técnicas, reconocidas como buenas prácticas, no son suficientes para gestionar eficientemente proyectos, para lo cual también se requirieren destrezas específicas y habilidades generales (PMI, 2017a). Esta combinación de elementos (habilidades, capacidades, pericia, experiencia, conocimientos y destrezas) relacionados con los contextos de trabajo se complementan e integran con una serie de atributos personales (actitud, comportamiento, motivación, personalidad y valores) (Omidvar et al., 2014).

Las competencias en dirección de proyectos movilizan conocimientos y habilidades para alcanzar un rendimiento esperado en el desempeño de un determinado trabajo (Takey y Carvalho, 2015). El desarrollo de estas, mediante entrenamiento, formación y/o mentoría, permite mejorar el rendimiento de los proyectos emprendidos, gracias a una mayor motivación, mejor auto-organización y menor necesidad de control (Bushuyev y Wagner, 2014).

No obstante, casi todos los estándares en dirección de proyectos están orientados a procesos. Por el contrario, muy 
pocos están basados en competencias, los cuales definen las necesidades de las personas para un buen desempeño en entornos de proyectos (Vukomanović et al., 2016). Mientras que los primeros generalmente prescriben procedimientos y métodos, procurando asegurar que las organizaciones tengan un enfoque universal en dirección de proyectos, los segundos presentan un amplio espectro de conocimientos, habilidades y destrezas que las personas necesitan para realizar las tareas encomendadas (Chipulu et al., 2013). Estas competencias pueden agruparse en tres bloques:

- $\quad$ Para Cheng, Dainty y Moore (2005):

Ocupacional, comprensivo y actitudinal.

- $\quad$ Para Le Deist y Winterton (2005):

Social, funcional y cognitivo.

- $\quad$ Para Binkley, et al. (2010):

Vivencias, herramientas y pensamientos.

- $\quad$ Para Onisk (2011):

Conformidad, profesional y comportamiento.

- $\quad$ Para Omidvar, et al. (2014):

Contextual, laboral y personal.

De forma análoga, las asociaciones de dirección de proyectos más extendidas del mundo, tanto la International Project Management Association (en adelante IPMA), como el Project Management Institute (en adelante PMI), junto con la Organización Internacional de Estandarización (en adelante ISO) y la Unión Europea (en adelante EU), también orientan sus estándares fundacionales, cuerpos de conocimiento, metodologías, guías prácticas, líneas de base y marcos en torno a:

- $\quad$ Para la IPMA en su estándar ICB 4 (IPMA, 2015):

Perspectiva, práctica y personal.

- $\quad$ Para la EU en su estándar PM2 (EU, 2016):

Perspectiva, práctica y personal.

- $\quad$ Para el PMI, en su estándar PMBOK 6 (PMI, 2017a): Estrategia y negocio, técnica y liderazgo.

- $\quad$ Para el PMI, en su estándar PMCDF 3 (PMI, 2017b): Conocimiento, rendimiento y personal.

- $\quad$ Para la ISO en su estándar internacional 21500 (ISO, 2012): Contextual, técnica y de comportamiento.

Si los enfoques de las dos asociaciones profesionales más extendidas a nivel mundial, PMI e IPMA, se contrastan, se puede observar que, si bien tanto el PMBOK 6 como el PM-
CDF 3 del PMI se centran en el fin mismo, partiendo de la premisa de que las competencias de las personas tienen un efecto directo en el rendimiento de los proyectos que llevan a cabo, el planteamiento de la ICB 4 de la IPMA se centra en los medios mismos, para lo cual ofrece una serie de propuestas para el desarrollo individual de las personas, entre las que se incluye, la experimentación, la simulación y la gamificación.

\section{Metodología}

La metodología de investigación utilizada es el estudio de casos, presentando soluciones mediante el análisis de situaciones reales previamente elegidas. Con esto, se pretende encontrar evidencias de un fenómeno diferenciado de su universo y respuestas a preguntas en un escenario dado, de ahí que no sean verdades universales (Castro, 2010). Además, su calidad es medida de acuerdo a diversos criterios, tales como fiabilidad, validez u objetividad (Binda y Balbastre, 2013).

Mediante el estudio de casos, se analiza cómo la metodología LSP, en combinación con la metodología Design Thinking, concebida para desarrollar la innovación centrada en las personas, puede ofrecer una amplia gama de oportunidades de aplicación exitosa en contextos en los que se dirigen y gestionan proyectos, destacando el enfoque basado en la adquisición y mejora de las competencias de las personas involucradas.

\subsection{Design Thinking}

El pensamiento creativo resuelve problemas, reduce riesgos y aumenta las posibilidades de abordar proyectos con éxito, al centrarse en las necesidades humanas, conectando conocimientos y experiencias desde múltiples disciplinas mediante la observación, y alcanzando soluciones deseables, económicamente viables y técnicamente factibles (Serrano y Blázquez, 2015). Esta resolución de retos se afronta de un modo creativo, recopilando información, analizando contenidos y proponiendo soluciones, desde la imaginación, gracias a la participación, y a través de la experimentación y prototipado (Ruiz et al., 2015). Los procesos de diseño están compuestos por una primera fase creativa, en la que se pregunta, propone, plantea y encuentra inspiración para crear soluciones alternativas, y por una segunda fase lógica, en la que se sintetizan los hallazgos, evalúa, desarrolla, prueba y ejecuta una solución final. En este contexto, si bien la creatividad combina de forma original conocimientos antes desconectados, para que esta tenga una utilidad práctica, es necesario incluir orden y estructura en el proceso (Morcillo y Alcachud, 2005). 
La metodología Design Thinking se desarrolla poniendo en práctica una serie de pasos, que quedan sintetizados en la Figura 2. Asimismo, presenta 4 características fundamentales (Beckman y Barry, 2007):
- $\quad$ Construcción de prototipos, consolidando la validación interna previa a la verificación.

- $\quad$ Contenido visual, fomentando la creatividad.

- Empatía, vía observación e interactuación.

- Trabajo en equipo, favoreciendo la contribución de todos los participantes.

Figura 2 Pasos de la metodología Design Thinking

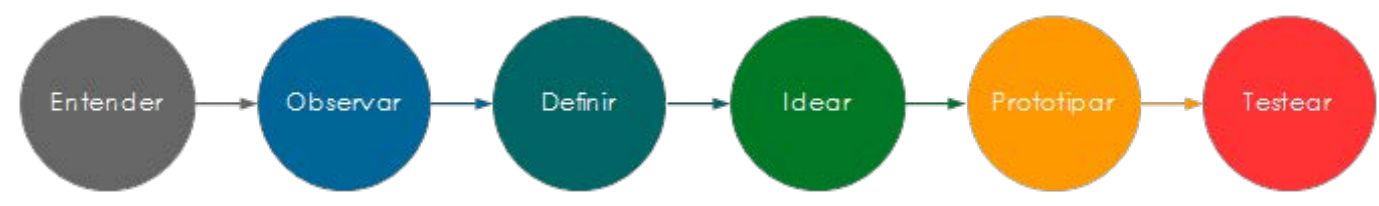

\subsection{Lego Serious Play ${ }^{\circledR}$}

La metodología LSP sigue un proceso secuencial en la que, mediante preguntas, los participantes van profundizando en la temática a lo largo de la sesión. Para ello, se van desarrollando ideas, proponiendo modelos, planteando esce- narios, etc., mediante la utilización de piezas de construcción de LegoTM, que sirven como base para una posterior toma de decisiones, intercambio de conocimientos y experiencias, así como un debate grupal mediante puesta en común. La metodología original desarrollada por sus creadores se basa en 4 pasos básicos, como representa la Figura 3.
Figura 3 Pasos nucleares de la metodología Lego Serious Play®

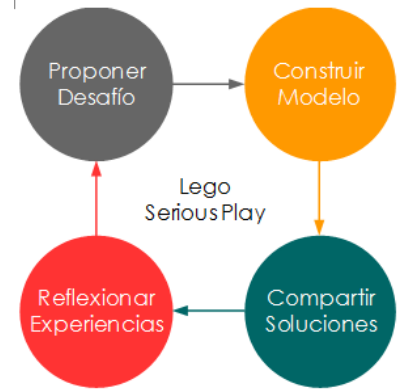

A partir de estos pasos, los creadores proponen 7 técnicas o estrategias para su puesta en práctica: construcción de modelos individuales, construcción de modelos compartidos, creación de escenarios, identificación de conexiones entre modelos, construcción de un sistema, simulación de una situación para la toma de decisiones y desarrollo de conclusiones.

\subsection{Evaluación del aprendizaje}

Para la evaluación del aprendizaje se opta por una variante del modelo de Kirkpatrick (Gresse von Wangenheim, Savi y Ferreti, 2012; Petri y Gresse von Wangenheim, 2016), utilizando el primero de sus 4 niveles: reacción, aprendizaje, comportamiento y resultados. Después de cada sesión, se difunde un cuestionario, mostrado en la Tabla A1-1 del Anexo
1. Un facilitador hace una pregunta abierta, en la que plantea un tema a tratar a los participantes, sin influir en la respuesta

2. Cada participante (o grupo) construye un modelo libre respondiendo a la pregunta anteriormente enunciada

3. Cada participante (o grupo) comparte lo que significa el modelo construido, con una historia que responde la pregunta

4. Cada participante (o grupo) internaliza y asienta la historia, reflexionando sobre lo visto y oído

1, lo que permite medir variables cualitativas (valoraciones, expectativas, emociones o motivaciones). La variación del modelo primigenio (Kirkpatrick y Kirkpatrick, 2006) se basa en una descomposición jerárquica del objetivo de evaluación (en motivación, experiencia y aprendizaje), siguiendo la métrica pregunta-objetivo (Caldiera y Rombach, 1994), como indica la Figura 4. A su vez, la motivación considera 4 categorías: atención, relevancia, confianza y satisfacción (Keller, 1987), la experiencia del juego considera pensamientos, sentimientos, placer y otras interacciones (Albert y Tullis, 2013) e incluye 5 dimensiones (inmersión, reto, competencia, diversión e interacción) (Calvillo 2009; Sweetser and Wyeth, 2005) y, por último, el aprendizaje observa 3 componentes de la taxonomía (Anderson et al., 2001) y 2 dimensiones adicionales sobre el aprendizaje a corto y largo plazo (Moody y Sindre, 2003). 
Figura 4 Modelo de evaluación del juego

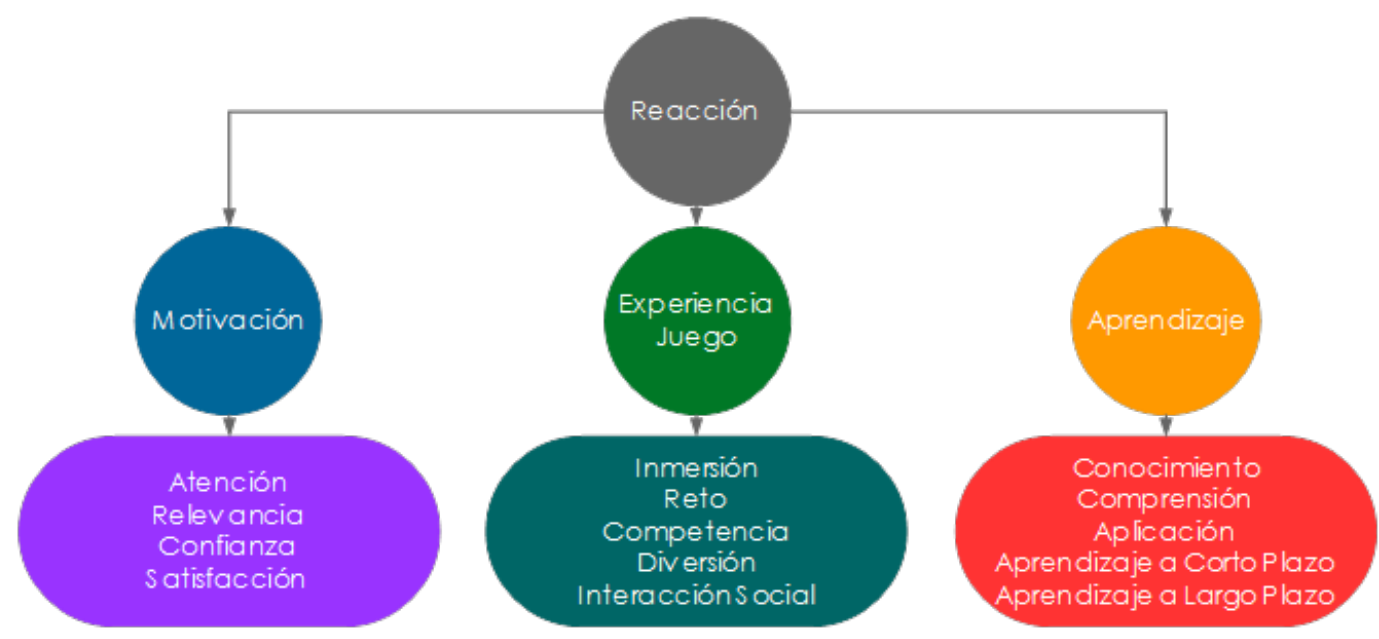

\section{Casos de estudio}

La metodología LSP ayuda a reflexionar y discutir ideas complejas, mejora el compromiso de los participantes e incrementa su capacidad de resolver problemas, razón por la que es utilizada como mecanismo para la creación de equipos y promoción de la creatividad, como resume la Figu-

ra 5, con resultados satisfactorios tanto en ámbitos empresariales (Albors et al. 2014) como industriales (Grienitz y Schmidt, 2012; Schulz et al., 2015), civiles (Bulmer, 2011), mecatrónicos (Mabogunje et al., 2008; Compos et al., 2012), sociológicos (Villamizar y González, 2015) y tecnológicos (Hyvönen, 2014; Kurkovsky, 2015), entre otros.

Figura 5 Marco de referencia de Lego Serious Play ${ }^{\circledR}$.

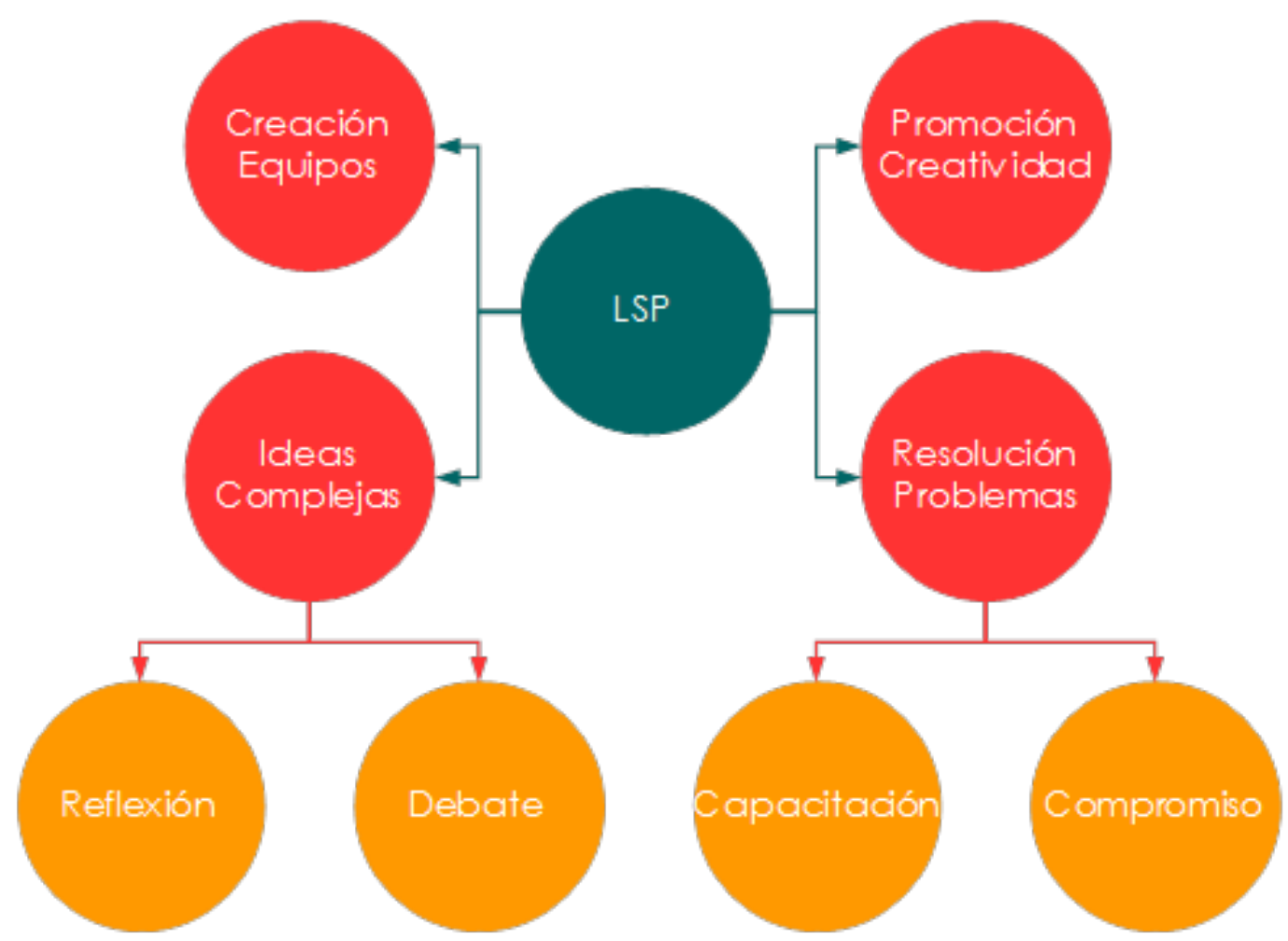


La dirección de proyectos es la disciplina encargada de su planteamiento y supervisión, para lo que organiza, administra y moviliza los recursos necesarios disponibles con el objeto de alcanzar los objetivos propuestos y cumplir las necesidades y expectativas de los interesados, generando valor mediante la entrega al cliente de los productos y/o servicios resultantes. Para lograr los rendimientos esperados y satisfacer a los involucrados, es ineludible contar con un director que ejerza su liderazgo, tenga una actitud abierta, sea fiable, practique una conducta ética, y posea habilidades de negociación, y con un equipo de trabajo comprometido y motivado, que trabaje en común, sea profesional, se oriente a resultados, y se vaya siendo empoderado a medida que aumenta su madurez (Cerezo-Narváez et al., 2016).

Las competencias en dirección de proyectos resultan esenciales en el mundo laboral del siglo XXI (Cerezo-Narváez et al., 2018), si bien pueden ser entrenadas mediante técnicas de gamificación (IPMA, 2015). En este contexto, LSP conecta la metodología Design Thinking con la dirección de proyectos, trabajando las competencias de las personas. No obstante, un aspecto clave en la aplicación de juegos serios para la impartición de contenidos es el análisis sobre su efectividad con fines académicos. Por ello, sus principales objetivos se centran en motivar para estudiar y promover el aprendizaje de los contenidos establecidos mediante actividades basadas en técnicas de gamificación.

En este artículo se estudian 2 casos de estudio, en los que se llevan a cabo actividades tanto individuales como grupales (en equipos) y colectivas encaminadas a trabajar en proyectos mediante técnicas de gamificación para la construcción de modelos tridimensionales. El primer caso de estudio, Caso 1, se inicia en la Universidad de Sevilla con la impartición de unos talleres orientados a trabajar en el inicio de los proyectos, abordando la constitución del proyecto, como primer paso, y la identificación de interesados a continuación. Por su parte, el segundo caso de estudio, Caso 2, comienza en la Universidad de Cádiz con el desarrollo de unas sesiones de trabajo planteadas para empezar la planificación de los proyectos, una vez estos se han iniciado y los interesados, incluyendo al equipo, han sido identificados y priorizados, mediante la recopilación de requisitos, tanto atributos y especificaciones como requerimientos de gestión.

\subsection{Caso 1: Constitución del proyecto e identi- ficación de interesados}

A partir del curso 2015-2016 en la Universidad de Sevilla, se pone en práctica en varias asignaturas de dirección de proyectos impartidas por el Departamento de Organización
Industrial y Gestión de Empresas II el desarrollo de talleres basados en la metodología LSP, dentro de las asignaturas de dirección de proyectos en el grado de Biotecnología, mención Biotecnología, así como en los másteres universitarios de ingeniería química e ingeniería de organización industrial. Este taller se ha repetido desde su inicio en 8 ocasiones, con un total de 115 participantes.

La idea base de este caso es desarrollar la constitución del proyecto abordando las etapas previas a la planificación. Tanto la norma ISO 21500 (ISO, 2012), como la guía de fundamentos para la dirección de proyectos (en adelante PMBOK $\left.{ }^{\circledR}\right)(P M I, 2017 a)$ señalan la identificación de oportunidades dentro del inicio del proyecto, el primero de los 5 grupos de procesos (inicio, planificación, implementación, control y cierre) en los que agrupan los procesos de la gestión de proyectos.

En este caso, la aplicación de la metodología LSP se centra en los dos procesos comunes del inicio del proyecto: constitución del proyecto e identificación de interesados. Además, permite el entrenamiento de un numeroso grupo de competencias, especialmente orientadas a las personas y a la práctica en dirección de proyectos. El taller se desarrolla en las primeras partes de la asignatura, lo que permite despertar interés en los alumnos por una materia que no conocen y generar una base, un proyecto que dé continuidad al aprendizaje de esta asignatura, mejorar la sintonía alumno-profesor e ir introduciendo conceptos, terminología, etc. de una forma amena.

Para cualquier proyecto es interesante comenzar por saber si resulta interesante y/o viable antes de continuar. Con este taller se identifican y evalúan los aspectos que determinan esta viabilidad. Asimismo, sirve para identificar las suposiciones, restricciones e hipótesis del proyecto, así como para establecer alternativas (Montero, 2017). En ciertos casos, la aplicación del método puede identificar al director del proyecto y a su equipo y establecer sus responsabilidades. A partir de la identificación del proyecto a realizar, LSP permite desarrollar la constitución de forma fluida, comenzando a trabajar para conocer cuál es el alcance del proyecto, principales entregables, hitos y riesgos que deben considerarse. Por último, el taller también incluye la identificación de los principales interesados en el proyecto, sus roles y cuál es su relación con el proyecto.

El desarrollo de este taller implica una sesión de 1,5-2 horas, aunque puede demorarse si se profundiza en el tema tratado durante la etapa de puesta en común. La distribución de estos talleres sigue el esquema presentado en la Tabla 1. 
Tabla 1 Distribución de una sesión para el Caso 1

\begin{tabular}{clc}
\hline Id. & \multicolumn{1}{c}{ Actividad (Etapa) } & Duración \\
\hline 1 & $\begin{array}{l}\text { Presentación de la metodología y con- } \\
\text { ceptos básicos }\end{array}$ & 15 min. \\
\hline 2 & $\begin{array}{l}\text { Construcción de los modelos individual- } \\
\text { mente }\end{array}$ & 20 min. \\
\hline 3 & $\begin{array}{l}\text { Presentación de cada modelo (idea de } \\
\text { proyecto) }\end{array}$ & 30 min. \\
\hline 4 & Reflexión conjunta & 15 min. \\
\hline 5 & Valoración de puntos tratados & 30 min. \\
\hline
\end{tabular}

Para el desarrollo de la actividad, en primer lugar, se describe en qué consiste la metodología y los conceptos básicos tanto de la constitución de un proyecto como de la identificación de los interesados. Se divide la clase en grupos de no más de 12 personas, a efectos logísticos, porque van a gestionar las piezas disponibles y, en menor manera, comunicar y relacionarse. En la experiencia del juego en que se aplica, el enfoque inicial en diferentes escenarios está orientado al análisis de factibilidad y al desarrollo del acta de constitución del proyecto. En este sentido, las preguntas abiertas son ¿Cuál es el proyecto?, ¿Quiénes son los interesados?, ¿Cuáles son los roles del equipo? y ¿Qué riesgos pueden aparecer?
A partir de la respuesta a estas preguntas, los equipos deben desarrollar la constitución del proyecto. Esto significa construir metáforas individuales para analizar las diferentes perspectivas del proyecto. Posteriormente, se analizan los puntos comunes y las divergencias en la idea del proyecto. A continuación, y en grupo, la metodología permite crear el escenario base, así como esbozar el alcance del proyecto. En una segunda etapa, cada alumno debe seleccionar mini-figuras para insertar en el escenario, que representen a los principales grupos de interés, de cara a empezar a identificar sus expectativas e intereses y cómo van a influir en el proyecto. De esta forma, el flujo de trabajo se alinea perfectamente con el inicio del proyecto. La Figura 6 muestra una sesión de aplicación:
Figura 6 Sesión de Lego Serious Play ${ }^{\circledR}$ para la iniciación del proyecto

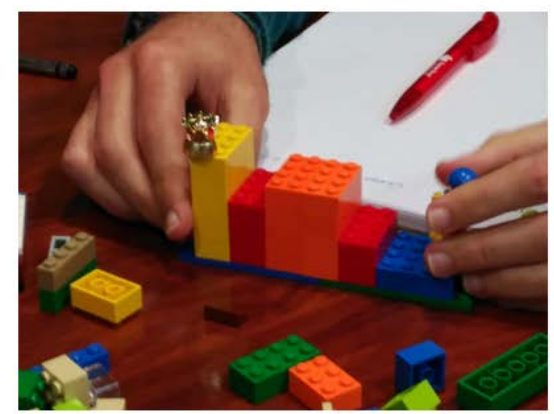

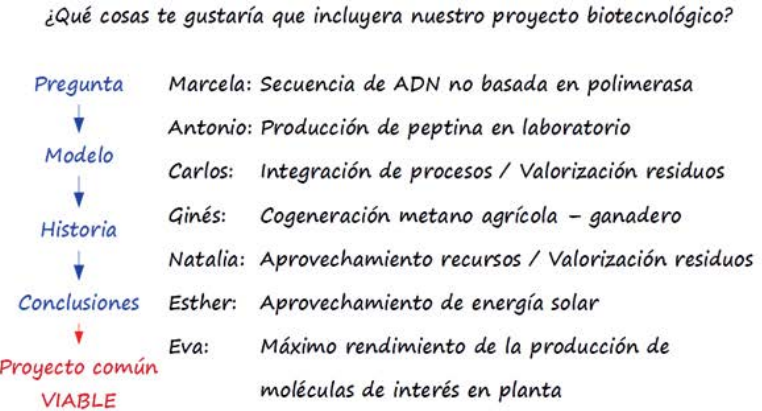

sacar conclusiones. Los resultados obtenidos son muy diversos. Algunos ejemplos se pueden ver en la Figura 7
El siguiente paso es comenzar a construir el proyecto (sistema), simular situaciones $y$, finalmente, tomar decisiones y
Figura 7 Ejemplos de modelos desarrollados en los talleres para la constitución del proyecto
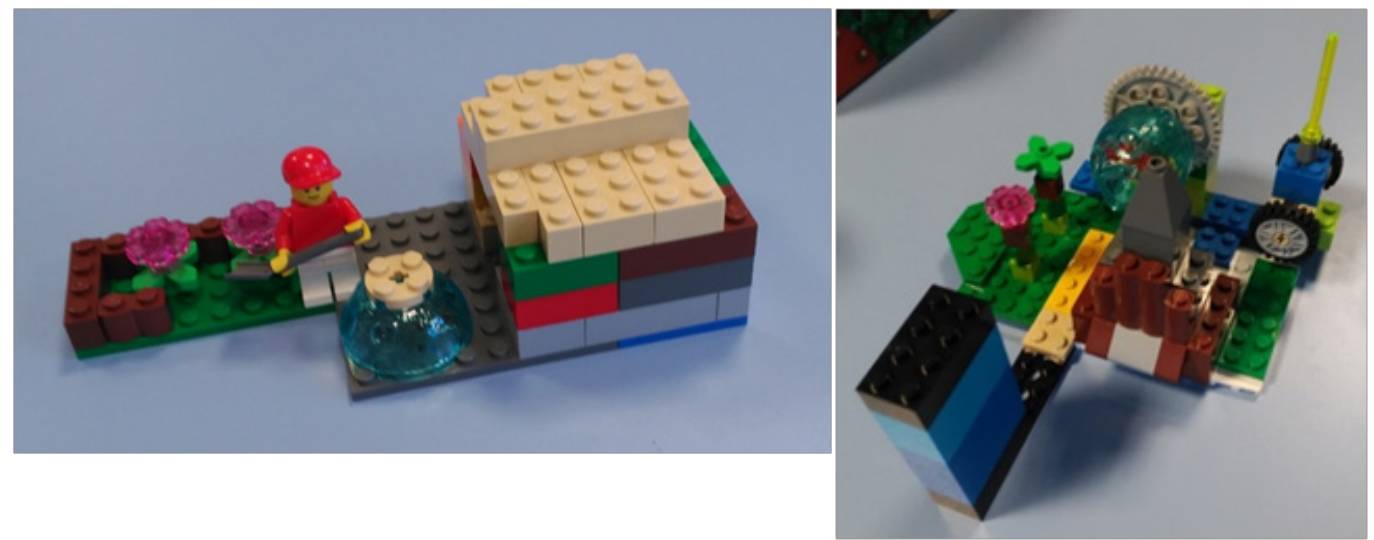
Como complemento a la dinámica realizada, se solicita a los alumnos que valoren si la herramienta les ha permitido extraer conclusiones sobre puntos básicos de la constitución del proyecto, tales como los puntos fuertes y débiles planteados, coherencia y concreción del alcance propuesto, claridad de los objetivos, existencia de una previsión de plazos y costes para su desarrollo, identificación e influencia de los principales interesados y recopilación de riesgos y oportunidades asociadas. Sobre estos puntos de reflexión se aplica una valoración con una escala tipo Likert de 5 opciones, que permita valorar el grado de acuerdo sobre estos puntos. Esta valoración persigue un doble objetivo:
1. Refuerzo del análisis inicial que requiere el proyecto y consolidación del aprendizaje de los conceptos

2. Extracción de los resultados estadísticos sobre el aprendizaje

Para el tamaño de la muestra obtenida con los 115 participantes, gracias a la homogeneidad de las respuestas recibidas y para un nivel de confianza del $95 \%$, se logra que el margen de error sea del 4,7\%, inferior al 5\% recomendado para este tipo de cuestionarios (Lenth, 2001). Los resultados obtenidos se muestran a continuación en la Tabla 2.

Tabla 2 Análisis de las reflexiones sobre los puntos tratados en el Caso 1

\begin{tabular}{|c|c|c|c|c|c|c|}
\hline & $\begin{array}{c}\text { Totalmente } \\
\text { en desacu- } \\
\text { erdo }\end{array}$ & $\begin{array}{l}\text { Bastante en } \\
\text { desacuerdo }\end{array}$ & $\begin{array}{l}\text { Ni en desac- } \\
\text { uerdo ni de } \\
\text { acuerdo }\end{array}$ & $\begin{array}{c}\text { Bastante de } \\
\text { acuerdo }\end{array}$ & $\begin{array}{l}\text { Totalmente } \\
\text { de acuerdo }\end{array}$ & NS/NC \\
\hline $\begin{array}{l}\text { Identificación } \\
\text { de fortalezas y } \\
\text { debilidades }\end{array}$ & $0 \%$ & $3 \%$ & $17 \%$ & $46 \%$ & $30 \%$ & $3 \%$ \\
\hline $\begin{array}{l}\text { Alcance co- } \\
\text { herente y con- } \\
\text { creto }\end{array}$ & $0 \%$ & $5 \%$ & $16 \%$ & $46 \%$ & $33 \%$ & $0 \%$ \\
\hline $\begin{array}{l}\text { O b j e t i v o s } \\
\text { claros }\end{array}$ & $0 \%$ & $0 \%$ & $29 \%$ & $36 \%$ & $35 \%$ & $0 \%$ \\
\hline $\begin{array}{l}\text { Identificación } \\
\text { de límites y re- } \\
\text { stricciones }\end{array}$ & $0 \%$ & $6 \%$ & $13 \%$ & $35 \%$ & $44 \%$ & $2 \%$ \\
\hline $\begin{array}{l}\text { Existencia de } \\
\text { una previsión } \\
\text { de plazos }\end{array}$ & $3 \%$ & $5 \%$ & $20 \%$ & $33 \%$ & $37 \%$ & $2 \%$ \\
\hline $\begin{array}{l}\text { Existencia de } \\
\text { una previsión } \\
\text { de costes }\end{array}$ & $0 \%$ & $7 \%$ & $40 \%$ & $33 \%$ & $20 \%$ & $0 \%$ \\
\hline $\begin{array}{l}\text { Conocimiento } \\
\text { de los intere- } \\
\text { sados }\end{array}$ & $0 \%$ & $0 \%$ & $8 \%$ & $57 \%$ & $35 \%$ & $0 \%$ \\
\hline $\begin{array}{l}\text { Identificación } \\
\text { de los riesgos }\end{array}$ & $0 \%$ & $6 \%$ & $17 \%$ & $32 \%$ & $43 \%$ & $2 \%$ \\
\hline
\end{tabular}

Para finalizar, se lleva a cabo una puesta en común posterior, en la que se discuten aquellas cuestiones que a los alumnos no les quedan claras o bien que se consideran en desacuerdo, parcial o totalmente, lo que permite generar debate y mejorar el aprendizaje de los mismos.

\subsection{Caso 2: Gestión de requisitos}

El Área de Proyectos de Ingeniería de la Universidad de Cádiz, durante el curso académico 2017-2018, pone en marcha una iniciativa organizando una serie de talleres prácticos en los que intentan aplicar, como sintetiza la Figura 8, las metodologías de Design Thinking, Trabajo en Competencias y Gamificación vía Construcción, utilizando material de LegoTM, en las asignaturas de $4^{\circ}$ curso de los grados en los que tienen docencia asignada, como parte de la misma (Cerezo-Narváez y Portela-Núñez, 2017). Sin embargo, estos talleres tienen un doble objetivo: Si bien, por un lado, se entrena con los estudiantes una serie de competencias en dirección de proyectos, por el otro, se les hace partes interesadas de un proyecto real, interviniendo en el proceso de recopilación de atributos y especificaciones, iniciándolos en los procesos de participación para la gestión de requisitos. Las sesiones, de 2 horas de duración, constan de 3 actividades constructivas, las dos primeras individuales y la tercera, grupal. 
Figura 8 Marco conceptual del caso 2

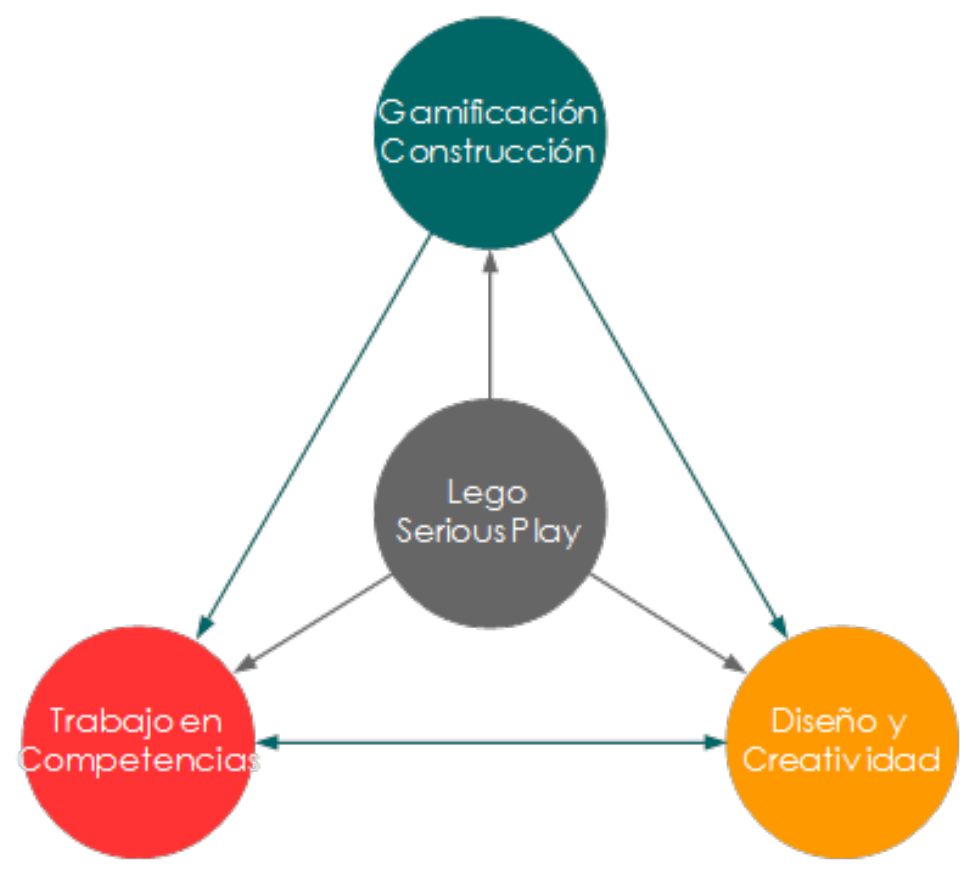

La primera actividad introductoria consiste en construir una torre, para introducir a los grupos en la dinámica de los talleres prácticos (planteamiento de reto, construcción, debate, puesta en común) y obtener su implicación. Para ello, han de construir un modelo que pueda ser considerado una torre, para lo que se divide al colectivo en dos mitades y se les da un plazo total de 5 minutos. Por un lado, las mesas impares, han de tener como base una ficha plana de color negro y como coronación una bandera de color verde. Para el resto del modelo, solo pueden usarse fichas de color amarillo y verde. Por otro lado, las mesas pares han de tener como base 2 fichas en forma de arco de color gris y como coronación una flor de color rosa, no pudiendo usar fichas de color amarillo ni verde para el resto del modelo, como muestra la Figura 9. En este primer ejercicio, se entrenan diversas competencias en dirección de proyectos, tanto específicas como sociales y contextuales. Asimismo, se debate sobre el cumplimiento o incumplimiento de diversos requisitos (como posibles demoras, uso de piezas no permitidas, resultados más anchos y/o largos que altos, discrepancias en base y/o coronación, etc.).
Figura 9 Resultados de la primera actividad "Torre” del Caso 2
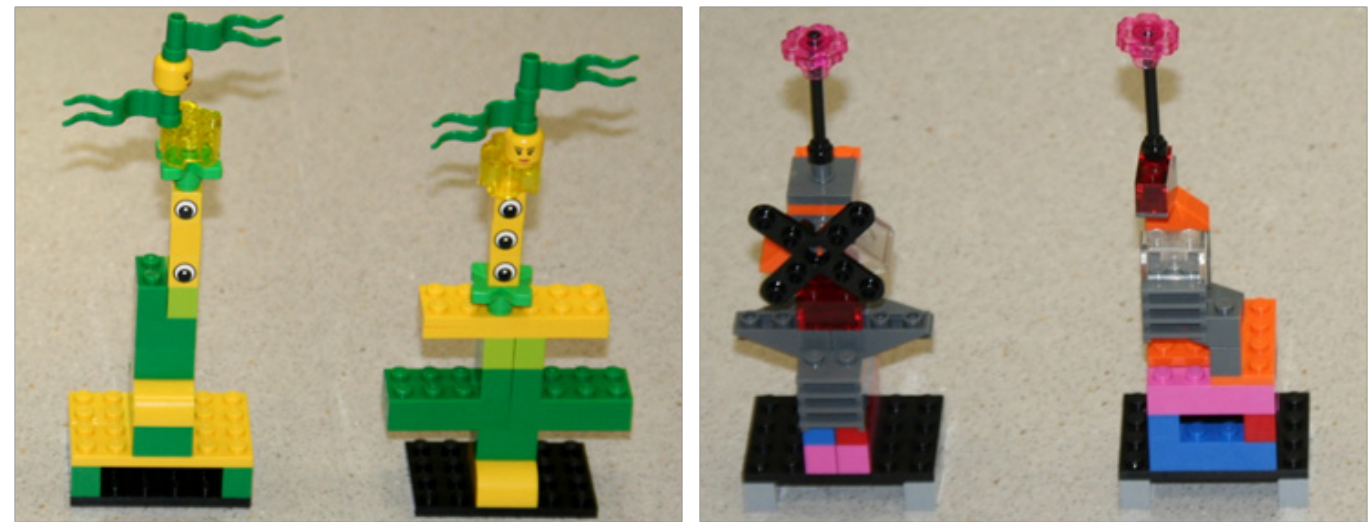
La segunda actividad introductoria consiste en reproducir un modelo prediseñado del que se adjunta únicamente una vista superior, representada en la Figura 10, habiéndose de suponer e interpretar la información no proporcionada. La construcción solicitada representa un parque-mirador en un entorno natural, para lo que se dispone de un tiempo total de ejecución de 10 minutos. Una vez ha transcurrido la mitad del tiempo (5 minutos), se suministra al grupo información adicional crucial para la correcta resolución del problema: El mirador ha de usar únicamente las fichas permitidas a las mesas impares del ejercicio anterior (piezas verdes y amarillas, bandera verde y ficha plana negra), y además ha de utilizarlas todas.

Figura 10 Información suministrada en la segunda actividad "Mirador" (vista superior) del Caso 2

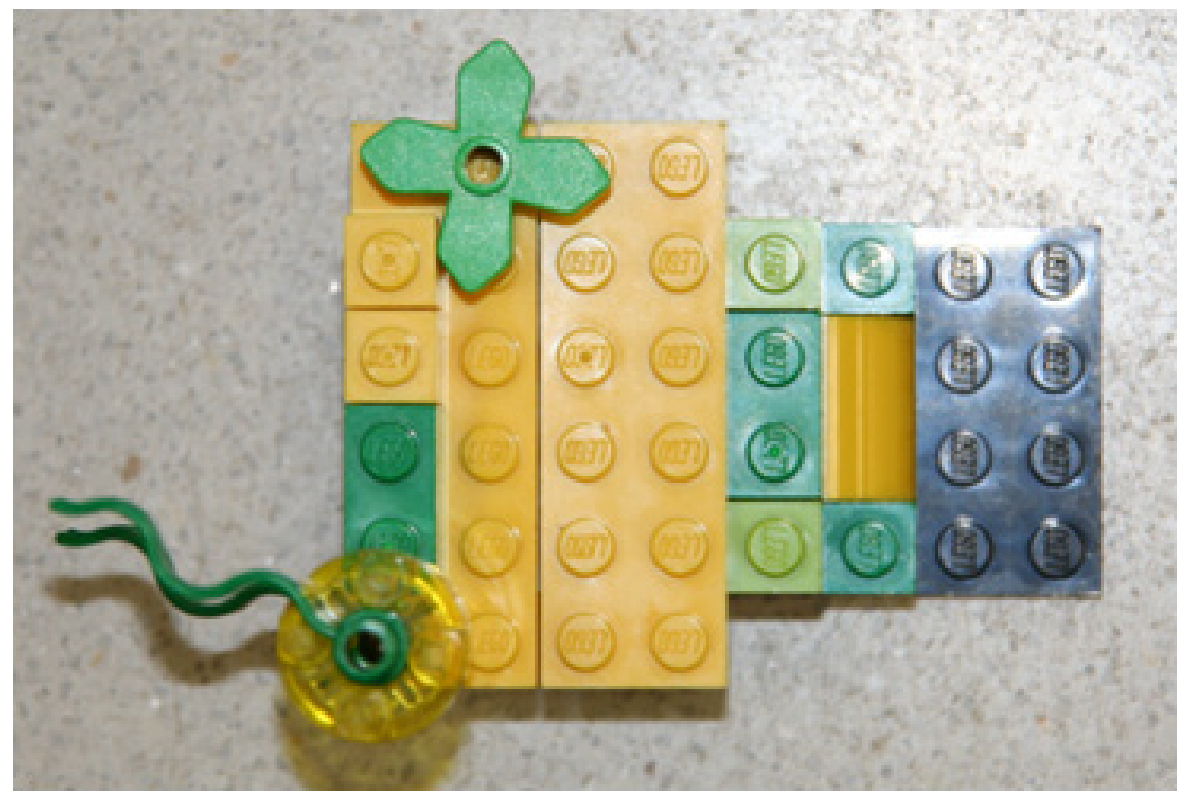

En este segundo ejercicio, se vuelven a entrenar diversas competencias en dirección de proyectos, tanto específicas como sociales y contextuales. El objetivo principal de este segundo ejercicio, tras haber roto el hielo en el primero, es resaltar la importancia de la documentación (gráfica) en los proyectos. Asimismo, gracias a la incorporación de nuevos requisitos una vez iniciado el proceso de construcción, se introduce el concepto de gestión de cambios, y de cómo estos pueden impactar, en menor o mayor medida, en las princi- pales líneas base del proyecto. Durante el período de puesta en común y reflexión posterior, se destaca la importancia de cuidar los detalles ( como orientar los ojos de las piezas amarillas hacia el paisaje) y de no incluir falsas suposiciones (como asociar el mástil de color negro, y por tanto no permitido, a la bandera de color verde). El resto de vistas del modelo, no suministradas en el enunciado, se presentan en la Figura 11.

Figura 11 Información no suministrada en la segunda actividad "Mirador" (vistas lateral y frontal) del Caso 2
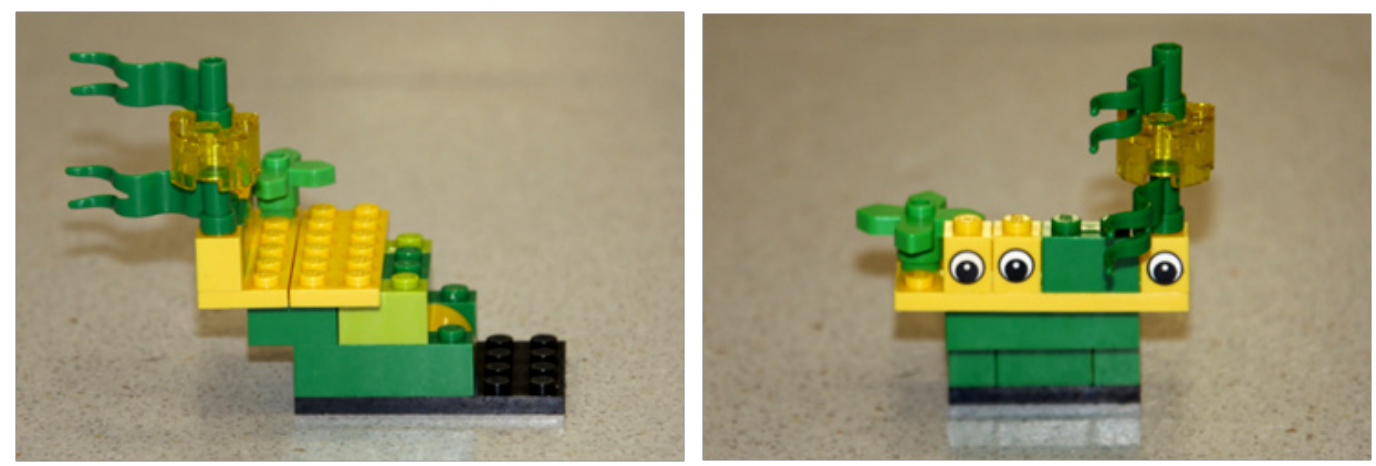
En la tercera actividad, se entrenan diversas competencias en dirección de proyectos, tanto específicas como sociales y contextuales, tal y como se ha mostrado anteriormente. Esta parte del taller es su núcleo central, consistente en diseñar un puente-pasarela que solvente la falta de conexión entre la estación ferroviaria de Las Aletas en Puerto Real y el Campus Universitario de Puerto Real de la Universidad de Cádiz (en adelante UCA), demandado por la comunidad universitaria del citado campus, cruzando la autovía CA-32 que conecta las localidades de El Puerto de Santa María y Puerto Real. Para ello, se solicita que los participantes se agrupen en equipos de 4 a 6 personas, concediéndoseles un primer plazo de 15 minutos para que se reúnan y tomen contacto con el set de piezas, se organicen para trabajar en equipo (eligiendo a una persona que ejerza de líder y repartiéndose los principales roles), lleven a cabo un diseño previo del modelo (teniendo en cuenta su escala, dimensión y proporción) y estimen las fichas que van a ser usadas, de acuerdo a los requisitos preliminares considerados (de resistencia, funcionalidad y apariencia).

A continuación, se les concede un plazo de 30 minutos para que construyan la maqueta del puente-pasarela, teniendo en cuenta que esta ha de soportar un peso de 1 kilogramo en el vano central, no se produzca una desviación superior a $\pm 25 \%$ en el número de piezas previstas y se cuide el diseño de la misma. Durante esta fase de construcción, los profesores actúan de facilitadores, aconsejando a los grupos, pero procurando no influir en las decisiones que, como equipos, han de ir tomando. Seguidamente, se establece un período de otros 15 minutos de debate y puesta en común acerca de las decisiones que se han ido tomando y, especialmente, de los requisitos que se han ido incorporando en el proyecto, desde 3 puntos de vista: constructivo, funcional y urbano. Las reflexiones acerca de los resultados obtenidos suelen versar en torno al tiempo que han soportado el peso exigido, a la resolución de los accesos al puente, a la inclusión de especificaciones singulares y modificaciones respecto del diseño previo a su construcción (como proporción, sistema estructural, material empleado, etc.). Además, y trascendiendo al propio taller, los profesores, actuando ahora como moderadores, van subrayando diversas conclusiones compartidas por los equipos en relación con cuestiones esenciales de la dirección de proyectos, como la gestión de requisitos, estructuración del trabajo, gestión de cambios, utilización de lecciones aprendidas, etc. Algunos de los resultados pueden observarse en la Figura 12.

Figura 12 Ejemplos de la segunda actividad "Puente-pasarela ESI/UCALas Aletas" del Caso 2
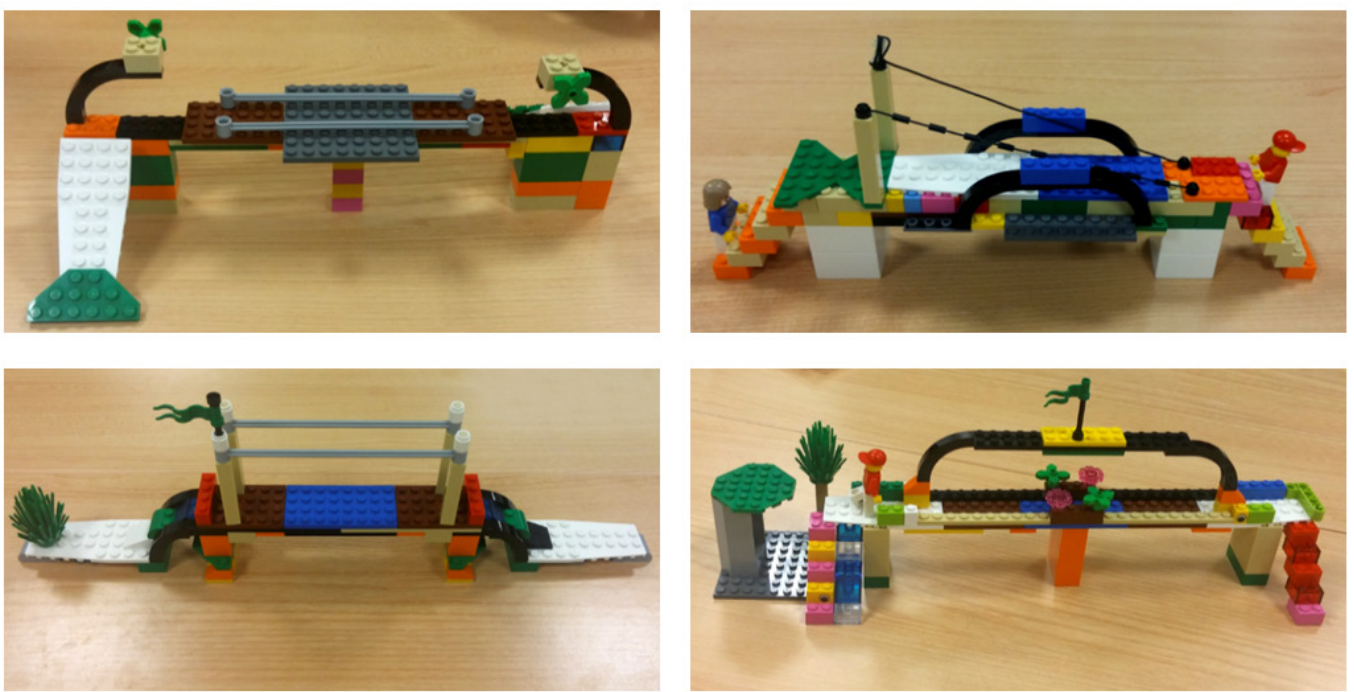

En la etapa de discusión y debate, se ponen en común los distintos requisitos considerados por los equipos, que quedan listados en la Tabla 3. Una vez recopilados los requisitos que podría llegar a incorporar el puente-pasarela, se utiliza como herramienta de ayuda a la decisión (de su incorporación) el método Kano (Kano et al., 1984), que está orientado a la evaluación de los requisitos de diseño de un producto, teniendo en cuenta 2 dimensiones y permitiendo identificar sus características primordiales. De esta manera, se logra discriminar los requisitos que son necesarios de los superfluos, haciendo distinción entre indiferentes, atractivos, unidimensionales y obligatorios 
Tabla 3 Recopilación jerarquizada de requisitos para el puente-pasarela vía LSP

\begin{tabular}{cccc}
\hline Código & Denominación & Código & Denominación \\
\hline 1 & Integración en el entorno & 8 & Ascensor \\
\hline 2 & Diseño innovador & 9 & Exposiciones itinerantes \\
\hline 3 & Cubrición & 10 & Climatización \\
\hline 4 & Insonorización & 11 & Iluminación automatizada \\
\hline 5 & Anti-vibración & 12 & Consumo sostenible \\
\hline & & 13 & Carril bici \\
\hline & Protección antivandalismo & 14 & Cobertura WiFi UCA \\
\hline
\end{tabular}

\section{Resultados}

El desarrollo completo de la aplicación de la metodología, que conduce a la clasificación de requisitos de cara a decidir acerca de su incorporación en el proyecto, queda incluido y definido paso a paso en el Anexo 2.
En la Tabla 4 se muestran las competencias trabajadas pos las actividades propuestas en ambos casos de estudio (Caso 1: idea, Caso 2: torre, mirador y puente), de acuerdo a la clasificación propuesta tanto por IPMA como por PMI. Nótese que el resto de competencias podrían ser aplicadas en otras actividades similares, cambiando solo algunos enfoques.

Tabla 4 Competencias entrenadas en las actividades desarrolladas.

${ }^{1}$ caso de estudio 1

${ }^{2}$ caso de estudio 2

\begin{tabular}{|c|c|c|c|c|c|}
\hline $\begin{array}{c}\text { PMI } \\
(2017 a, 2017 b)\end{array}$ & $\begin{array}{l}\text { IPMA } \\
\text { (2015) }\end{array}$ & Idea $^{1}$ & Torre $^{2}$ & Mirador $^{2}$ & Puente $^{2}$ \\
\hline $\begin{array}{l}\text { Gestión estratégica y de } \\
\text { negocio: }\end{array}$ & Perspectiva: & & & & \\
\hline Estrategia y negocio & Estrategia & & & & $\bullet$ \\
\hline Activos de los procesos & Gobernanza, estructuras y procesos & & & & \\
\hline Sistemas organizacionales & Estándares y regulaciones & $\bullet$ & $\bullet$ & $\bullet$ & $\bullet$ \\
\hline Política y poder & Poder e interés & & & & \\
\hline Factores ambientales & Cultura y valores & & & & \\
\hline Liderazgo: & Personal: & & & & \\
\hline Gerencia & Autorreflexión y autogestión & $\bullet$ & $\bullet$ & $\bullet$ & $\bullet$ \\
\hline Profesionalidad & Integridad personal y fiabilidad & & $\bullet$ & $\bullet$ & \\
\hline Asertividad & Comunicación personal & $\bullet$ & & & $\bullet$ \\
\hline Personalidad & Relaciones y participación & $\bullet$ & & & $\bullet$ \\
\hline Liderazgo & Liderazgo & & & & $\bullet$ \\
\hline Colaboración & Trabajo en equipo & & & & $\bullet$ \\
\hline
\end{tabular}




\begin{tabular}{|c|c|c|c|c|c|}
\hline Trato con las personas & Conflictos y crisis & & & & \\
\hline Habilidad cognitiva & Ingenio & $\bullet$ & $\bullet$ & $\bullet$ & $\bullet$ \\
\hline Obtención de resultados & Negociación & $\bullet$ & & & $\bullet$ \\
\hline Efectividad & Orientación a resultados & & $\bullet$ & $\bullet$ & $\bullet$ \\
\hline Téenica: & Práctica: & & & & \\
\hline Objetos del proyecto & Diseño del proyecto & $\bullet$ & & & $\bullet$ \\
\hline Metas y objetivos & Requisitos, objetivos y beneficios & $\bullet$ & $\bullet$ & $\bullet$ & $\bullet$ \\
\hline Alcance & Alcance & & $\bullet$ & $\bullet$ & $\bullet$ \\
\hline Cronograma & Tiempo & & $\bullet$ & $\bullet$ & $\bullet$ \\
\hline Comunicaciones & Organización e información & & & & $\bullet$ \\
\hline Calidad & Calidad & & & $\bullet$ & $\bullet$ \\
\hline Costos & Finanzas & & & & \\
\hline Recursos & Recursos & & $\bullet$ & $\bullet$ & $\bullet$ \\
\hline Adquisiciones & Aprovisionamiento y alianzas & & & & \\
\hline Programación & Planificación y control & & $\bullet$ & $\bullet$ & - \\
\hline Riesgos & Riesgo y oportunidad & & & & \\
\hline Interesados & Partes interesadas & $\bullet$ & & & e \\
\hline Integración & Cambio y transformación & & & $\bullet$ & \\
\hline
\end{tabular}

Los resultados de la aplicación del cuestionario basado en el modelo de Kirkpatrick, recopilado en la Tabla A1-1 del Anexo 1, en las experiencias desarrolladas tanto en el caso 1 como en el caso 2, se muestran en este apartado. En una visión general de los mismos se puede apreciar una valoración de la experiencia positiva, como se comprueba a continuación. La Tabla 5 muestra los resultados combinados de la evaluación de los distintos sub-elementos de cada una de las categorías.
Como se puede apreciar, los resultados son muy positivos en todos los ámbitos. Una gran mayoría, por encima del $80 \%$, está bastante de acuerdo o totalmente de acuerdo en las afirmaciones relacionadas con cada una de estas categorías. Para comprender mejor los resultados obtenidos, se van a mostrar en primer lugar cada una de las categorías de forma separada, con las valoraciones obtenidas para cada una de sus dimensiones correspondientes. 
Tabla 5 Tabla de frecuencias relativas de las dimensiones analizadas.

\begin{tabular}{|c|c|c|c|c|c|}
\hline Dimensión & $\begin{array}{c}\text { Totalmente en } \\
\text { desacuerdo }\end{array}$ & $\begin{array}{l}\text { Bastante en } \\
\text { desacuerdo }\end{array}$ & $\begin{array}{l}\text { Ni en desac- } \\
\text { uerdo ni de } \\
\text { acuerdo }\end{array}$ & $\begin{array}{l}\text { Bastante de } \\
\text { acuerdo }\end{array}$ & $\begin{array}{c}\text { Totalmente de } \\
\text { acuerdo }\end{array}$ \\
\hline Motivación & $1 \%$ & $3 \%$ & $10 \%$ & $27 \%$ & $59 \%$ \\
\hline $\begin{array}{l}\text { Experiencia de } \\
\text { juego }\end{array}$ & $1 \%$ & $2 \%$ & $10 \%$ & $27 \%$ & $60 \%$ \\
\hline Aprendizaje & $2 \%$ & $2 \%$ & $11 \%$ & $31 \%$ & $54 \%$ \\
\hline
\end{tabular}

La primera categoría tratada por el cuestionario considera la categoría de la motivación percibida por los participantes. La Tabla 6 muestra los valores obtenidos en cada dimensión de este elemento, que como se muestra, con excelentes valo- raciones. La segunda categoría analizada es la experiencia del juego, que también ofrece muy buenas valoraciones. La Tabla 7 muestra los valores obtenidos en cada dimensión.
Tabla 6 Frecuencias relativas de las dimensiones analizadas para la motivación.

\begin{tabular}{|c|c|c|c|c|c|}
\hline Dimensión & $\begin{array}{c}\text { Totalmente en } \\
\text { desacuerdo }\end{array}$ & $\begin{array}{l}\text { Bastante en } \\
\text { desacuerdo }\end{array}$ & $\begin{array}{l}\text { Ni en desacuer- } \\
\text { do ni de acuerdo }\end{array}$ & $\begin{array}{c}\text { Bastante de } \\
\text { acuerdo }\end{array}$ & $\begin{array}{c}\text { Totalmente de } \\
\text { acuerdo }\end{array}$ \\
\hline Atención & $0 \%$ & $0 \%$ & $7 \%$ & $38 \%$ & $55 \%$ \\
\hline Relevancia & $0 \%$ & $2 \%$ & $10 \%$ & $21 \%$ & $67 \%$ \\
\hline Confianza & $2 \%$ & $8 \%$ & $12 \%$ & $25 \%$ & $53 \%$ \\
\hline Satisfacción & $0 \%$ & $2 \%$ & $12 \%$ & $23 \%$ & $63 \%$ \\
\hline
\end{tabular}

Tabla 7 Frecuencias relativas de las dimensiones analizadas para experiencia del juego.

\begin{tabular}{lccccc}
\hline Dimensión & $\begin{array}{c}\text { Totalmente en } \\
\text { desacuerdo }\end{array}$ & $\begin{array}{c}\text { Bastante en } \\
\text { desacuerdo }\end{array}$ & $\begin{array}{c}\text { Ni en desacuer- } \\
\text { do ni de acuerdo }\end{array}$ & $\begin{array}{c}\text { Bastante de } \\
\text { acuerdo }\end{array}$ & $\begin{array}{c}\text { Totalmente de } \\
\text { acuerdo }\end{array}$ \\
\hline Inmersión & $1 \%$ & $4 \%$ & $17 \%$ & $24 \%$ & $54 \%$ \\
\hline Interacción social & $0 \%$ & $1 \%$ & $5 \%$ & $21 \%$ & $73 \%$ \\
\hline Reto & $1 \%$ & & & & $5 \%$ \\
\hline \\
Diversión
\end{tabular}

Por último, se analiza la valoración de los participantes en relación a su aprendizaje; igualmente con resultados muy positivos para cada dimensión, excepto en el caso del aprendizaje en el largo plazo, como muestra la Tabla 8.
Tabla 8 Frecuencias relativas de las dimensiones analizadas del aprendizaje.

\begin{tabular}{lccccc}
\hline Dimensión & $\begin{array}{c}\text { Totalmente en } \\
\text { desacuerdo }\end{array}$ & $\begin{array}{c}\text { Bastante en } \\
\text { desacuerdo }\end{array}$ & $\begin{array}{c}\text { Ni en desacuer- } \\
\text { do ni de acuerdo }\end{array}$ & $\begin{array}{c}\text { Bastante de } \\
\text { acuerdo }\end{array}$ & $\begin{array}{c}\text { Totalmente de } \\
\text { acuerdo }\end{array}$ \\
\hline Competencia & $0 \%$ & $2 \%$ & $9 \%$ & $35 \%$ & $54 \%$ \\
\hline $\begin{array}{l}\text { Aprendizaje corto } \\
\text { plazo }\end{array}$ & $0 \%$ & $2 \%$ & $10 \%$ & $31 \%$ & $57 \%$ \\
\hline $\begin{array}{l}\text { Aprendizaje largo } \\
\text { plazo }\end{array}$ & $10 \%$ & $7 \%$ & $17 \%$ & $32 \%$ & $34 \%$ \\
\hline
\end{tabular}


Representando la valoración obtenida para cada una de las dimensiones analizadas, como resume la Figura 13, se puede apreciar que en todos los casos el grado de acuerdo es mayoritario. Además, en 26 de las 28 variables consideradas, este supera el $80 \%$ y solo en el caso de la dimensión del aprendizaje a largo plazo existe desacuerdo. Dentro de una generalidad de altas valoraciones, las dimensiones mejor posicionadas, considerando las dos opciones de acuerdo y totalmente de acuerdo, son: atención, interacción social y diversión (superando el 90\%), seguidas muy de cerca por relevancia, competencia y aprendizaje a corto plazo.

Figura 13 Visión global de la evaluación del aprendizaje.

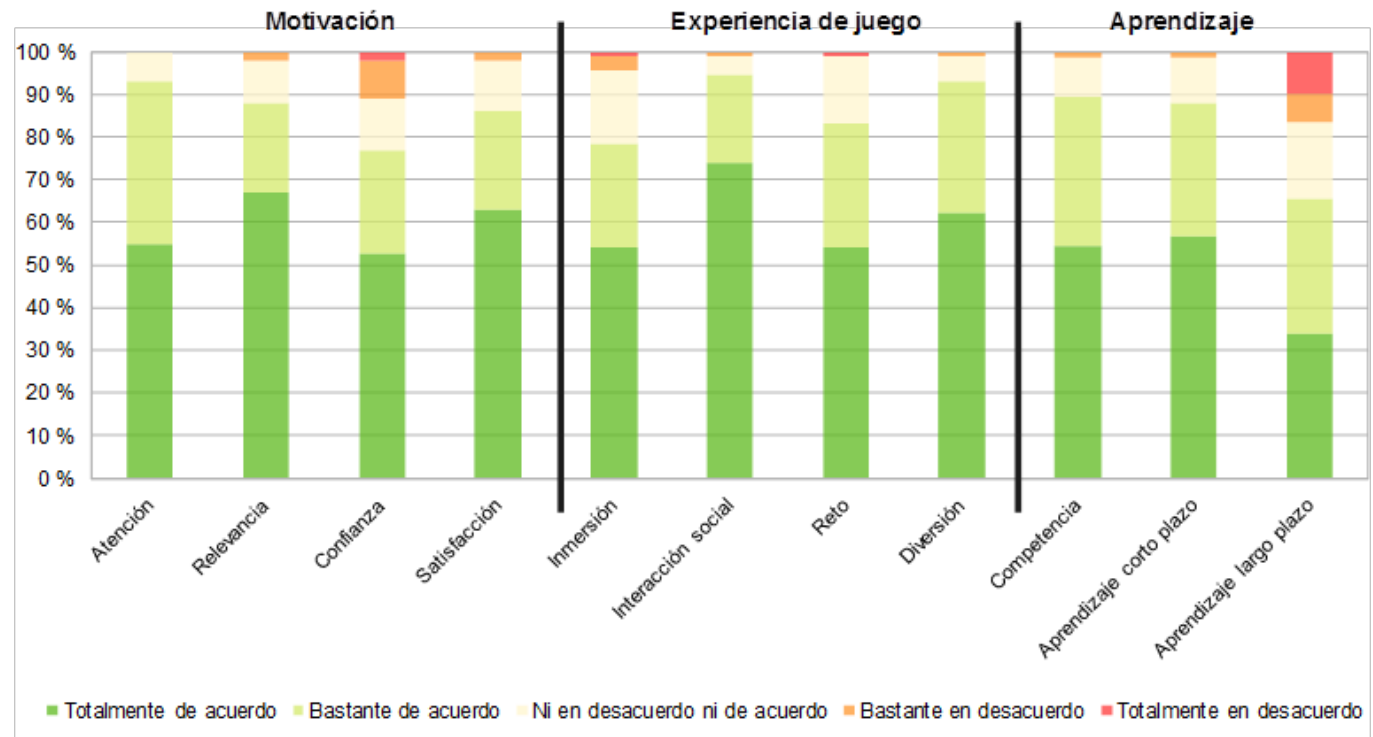

Atendiendo a las distintas dimensiones a nivel individual analizadas en cada categoría, se pueden considerar resultados más detallados. En el apartado de motivación, destaca la variable de contenido, seguido como aspectos mejor valorados por los alumnos el diseño, la variedad y el esfuerzo. La variable que obtiene peor valoración, la facilidad. En la categoría de experiencia de juego, destaca la interacción y se valoran por encima del $80 \%$ de acuerdo las dimensiones de tiempo, diversión, cooperación/competencia y reto. Por último, en el caso de aprendizaje, solo existe una variable con acuerdo superior al $80 \%$; que es la eficacia. Por el contrario, la variable que muestra algún desacuerdo (si bien menor al $10 \%$ ) es la eficiencia.

\section{Conclusiones}

La combinación de las metodologías de Design Thinking, Trabajo en Competencias (en Dirección de Proyectos) y Gamificación (vía Construcción) en los talleres de los 2 casos de estudio propicia una gran receptividad, lo que hace que los participantes se comporten respetuosamente, estén motivados, se involucren en los ejercicios planteados y disfruten, con relajación y sentido del humor, como se deduce en los resultados obtenidos tras aplicar el cuestionario del Anexo 1.

El ambiente lúdico fomenta la comunicación y participación, dando la oportunidad de expresar puntos de vista propios, sin ser influenciados por el resto del grupo, eliminando obstáculos discriminatorios, culturales o de género, desarrollando la conciencia de pertenencia a un colectivo, y poniendo en práctica capacidades de colaboración y creatividad, al navegar por su inteligencia integrada (visual, espacial, lingüística y cinestésica) construyendo modelos representativos simples como respuesta a problemas reales planteados y desarrollando numerosas habilidades transversales (tales como actitud abierta, cooperación, compromiso, motivación, resiliencia, trabajo en equipo, etc.).

La dirección de proyectos, gracias a su carácter transversal y globalizador, permite fortalecer y ampliar los conocimientos y habilidades de los participantes de los talleres. El establecimiento de objetivos grupales, la concatenación de procesos técnicos y de gestión y la aplicación de habilidades interpersonales, permite hacer entender que los proyectos solo pueden ser abordados desde una perspectiva colectiva, en la que el rendimiento depende no solo de los resultados individuales sino también de sus relaciones.

Con el apoyo de Lego Serious Play®, la iniciación del proyecto, a través de la constitución del proyecto y de la identificación de interesados, así como la gestión de interesados puede entrenarse fácilmente con la metodología expuesta, de manera que se enfoca y sirve de guía para alcanzar los objetivos, estudiando de manera preliminar cómo afrontar los modelos tridimensionales y finalizando las construcciones cumpliendo supuestos y restricciones, aunque fomentando la creatividad para enriquecer los diseños iniciales y, finalmente, poniendo en común, debatiendo, propiciando una reflexión crítica sobre su manera de proceder y generando lecciones aprendidas. 
Los casos estudiados muestran que el uso de la metodología Lego Serious Play®, aislada o combinación con otras metodologías, como Design Thinking, aporta numerosas ventajas desde el punto de vista académico, al fomentar la participación, incrementar la motivación de los estudiantes, permitir introducir conceptos nuevos o complejos o consolidar los conceptos principales y permitir ponerlos en práctica. Asimismo, a partir de la evaluación realizada sobre la motivación, experiencia de juego y aprendizaje, destaca la valoración realizada por los participantes en los talleres prácticos en relación al contenido, diseño, interacción, tiempo, diversión y aprendizaje a corto plazo, entre otras.

Por otro lado, gracias al juego, se logra generar unas condiciones propicias para debatir la incorporación de una serie de especificaciones y atributos en un proyecto real por parte de los usuarios finales del mismo (pasando de jugadores a suministradores de requisitos). La motivación permite alcanzar un enfoque de partida óptimo y la creatividad sobrevenida al recrear escenarios tridimensionales, consigue que se consideren una serie de posibilidades antes inexploradas. A través de los métodos Kano y su variante analítica A-Kano, como queda descrito en el Anexo 2, se permite extraer más información de los usuarios, rompiendo con el pensamiento que vincula proporcionalmente la satisfacción de estos con las funcionalidades incluidas, agrupando las preferencias entre aquellas que generan entusiasmo, otras que son proporcionales (lineales), otras que son imprescindibles, otras que generan indiferencia e incluso otras opciones que causan rechazo.

\section{Referencias}

Albert, W. y Tullis, T. (2013). Measuring the user experience: Collecting, analyzing y presenting usability metrics. Morgan Kaufmann: Waltham.

Albors, J., de Miguel, M., de Miguel, B., Segarra, M.V. y Barrera, P. (2014). La herramienta Lego Serious Play®: Análisis de su uso en los estudios de Grado y Máster de la Facultad de ADE. En I Jornada de Investigación de la Facultad de Administración y Dirección de Empresas (pp. 43-48). Valencia: Universitat Politècnica de València.

Anderson, L.W., Krathwohl, D.R., Airasian, P., Cruikshank, K., Mayer, R., Pintrich, P., Wittrock, M. (2001). A taxonomy for learning, teaching and assessing: A revision of Bloom's taxonomy. Cognition and Instruction, 9(2), 137-175.

Beckman, S. L. y Barry, M. (2007). Innovation as a learning process: Embedding design thinking. California Management Review, 50(1), 25-56.
Berger, C., Blauth, R., Boger, D., Bolster, C., Burchill, G., DuMouchel, W., Walden, D. (1993). Kano's methods for understanding customer-defined quality. Center for Quality of Management Journal, 2(4), 2-36.

Binda, N.U. y Balbastre-Benavent, F. (2013). Investigación cuantitativa e investigación cualitativa: Buscando las ventajas de las diferentes metodologías de investigación. Revista de Ciencias Económicas, 31(2), 179-187.

Binkley, M., Erstad, O., Herman, J., Raizen, S. y Ripley, M. (2010). Defining 21st century skills. Draft White Paper 1. Melbourne.

Bonilla, J.B. (2008). La educación basada en competencias como instrumento de política educativa y laboral. Revista Mexicana de Agronegocios, XII(22), 490-502.

Building value with Lego: How to create and destroy taboos through collaboration. (2012). Strategic Direction, 28(9), 21-24. https://doi.org/10.1108/02580541211256512.

Bulmer, L. (2011). The use of Lego ${ }^{\circledR}$ Serious PlayTM in the engineering design classroom. En II Canadian Engineering Education Association Conference. https://doi. org/10.24908/pceea.v0i0.3699.

Bushuyev, S.D. y Wagner, R.F. (2014). IPMA Delta and IPMA organisational competence baseline (OCB). International Journal of Managing Projects in Business, 7(2), 302-310. https://doi.org/10.1108/IJMPB-10-2013-0049.

Caldiera, V. y Rombach, H.D. (1994). The goal question metric approach. Encyclopedia of Software Engineering, 2(1994), 528-532.

Calvillo, E.H. (2009). On the core elements of the experience of playing video games. University College London.

Castro, E. (2010). El estudio de casos como metodología de investigación científica en dirección y economía de la empresa. Revista Nacional de Administración, 1(2), 3154. https://doi.org/10.1016/S1135-2523(12)60033-1.

Cerezo-Narváez, A., Bastante-Ceca, M.J. y Yagüe-Blanco, J.L. (2018). Traceability of intra- and interpersonal skills: From education to labor market. En M. Otero-Mateo y A. Pastor-Fernández (Eds.), Human Capital and Competences in Project Management (pp. 87-110). Rijeka: InTech. https://doi.org/10.5772/intechopen.71275. 
Cerezo-Narváez, A., Pastor-Fernández, A., Otero-Mateo, M. y Portela-Núñez, J.M. (2016). Influencia de las competencias interpersonales en el camino hacia la madurez organizacional en dirección de proyectos. En XX Congreso Internacional de Dirección e Ingeniería de Proyectos (pp. 0028-0040). Cartagena: AEIPRO.

Cerezo-Narváez, A. y Portela-Núñez, J.-M. (2017). Introducción a proyectos de ingeniería con Lego Serious Play. En A. Pastor-Férnandez y J.L. Yagüe-Blanco (Eds.), Nuevas tendencias de enseñanza aprendizaje aplicadas a la formación en Dirección de Proyectos. (pp. 190-244). Cádiz: Universidad de Cádiz y AEIPRO.

Cheng, M.I., Dainty, A.R.J. y Moore, D.R. (2005). What makes a good project manager? Human Resource Management Journal, 15(1), 25-37. https://doi. org/10.1111/j.1748-8583.2005.tb00138.x.

Chipulu, M., Neoh, J.G., Ojiako, U. y Williams, T. (2013). A multidimensional analysis of project manager competences. IEEE Transactions on Engineering Management, 60(3), 506-517. https://doi.org/10.1109/ TEM.2012.2215330.

Compos, D., Lima, R.M. y Fernandes, J.M. (2012). Identification and asessment of behavioral competences in multidiscipline temas within design projects. En Proceedings of the Fourth International Symposium on Project Approaches in Engineering Education (pp. 15-22). São Paulo: Pontifical Catholic University of São Paulo.

EU (European Union). (2016). The PM2 project management methodology guide. Luxemburgo: Publications Office of the European Union. https://doi.org/10.2799/957700.

Fong, D. (1996). Using the self-stated importance questionnaire to interpret Kano questionnaire results. Center for Quality Management Journal, 5(3), 21-24.

Gresse von Wangenheim, C., Savi, R. y Ferreti, A. (2012). DELIVER!-An educational game for teaching earned value management in computing courses. Information and Software Technology, 54(3), 286-298. https://doi. org/10.1016/j.infsof.2011.10.005.

Grienitz, V. y Schmidt, A.M. (2012). Scenario workshops for strategic management with Lego Serious Play. Problems of Management in the 21st Century, 3, 26-35.

Hyvönen, J. (2014). Creating shared understanding with Lego Serious Play. En Proceedings of the Seminar 58314308 Data- and Value-Driven Software Engineering with Deep Customer Insight (pp. 36-42). Helsinki: University of Helsinki.
ISO (International Organization for Standardization). (2012). ISO 21500: Guidance on project management. Geneva: ISO.

IPMA (International Project Management Association). (2015). Individual competence baseline for project, programme y portfolio management ( $4^{\mathrm{a}}$ ed.). Zurich: IPMA.

Jensen, A.F., Thuesen, C. y Geraldi, J. (2016). The projectification of everything: Projects as a human condition. Project Management Journal, 47(3), 21-34.

Kano, N., Seraku, N., Takahashi, F. y Tsuji, S. (1984). Attractive quality and must-be quality. Journal of the Japanese Society for Quality Control, 14(2), 147-156. https://doi.org/10.20684/quality.14.2_147.

Keller, J.M. (1987). Development and use of the ARCS model of motivational design. Journal of Instructional Development, 10(1932), 2-10. https://doi.org/10.1002/ pfi.4160260802.

Kirkpatrick, D.L. y Kirkpatrick, J.D. (2006). Evaluating training programs: The four levels ( $3^{\mathrm{a}} \mathrm{ed}$.). San Francisco: McGraw-Hill Education.

Kristiansen, P., Hansen, P.K. y Nielsen, L.M. (2009). Articulation of tacit and complex knowledge. En 13 International Workshop of IFIP WG 5.7 SIG (pp. 77-86). Zurich: International Federation for Information Processing.

Kurkovsky, S. (2015). Teaching software engineering with LEGO Serious Play. En Proceedings of the 2015 ACM Conference on Innovation and Technology in Computer Science Education - ITiCSE '15 (pp. 213-218). New York: ACM Press. https://doi.org/10.1145/2729094.2742604.

Le Deist, F.D. y Winterton, J. (2005). What is competence? Human Resource Development International, 8(1), 2746. https://doi.org/10.1080/1367886042000338227.

Lego. (2010). Open-source / <Introduction to Lego ${ }^{\circledR}$ Serious Play ${ }^{\circledR}>$.

Lenth, R.V. (2001). Some practical guidelines for effective sample size determination. The American Statistician, 55(3), 187-193. https://doi. org/10.1198/000313001317098149.

Mabogunje, A., Hansen, P.K., Eris, O. y Leifer, L. (2008). Product design and intentional emergence facilitated by Serious Play. En Proceedings of 7th Norddesign Conference (pp. 9-18). Tallin: The Design Society. 
Matzler, K. y Hinterhuber, H.H. (1998). How to make product development projects more successful by integrating Kano's model of customer satisfaction into quality function deployment. Technovation, 18(1), 25-38. https:// doi.org/10.1016/S0166-4972(97)00072-2.

Mccusker, S. (2014). Lego Serious Play: Thinking about teaching and learning. International Journal of Knowledge, Innovation and Entrepreneurship, 2(1), 27-37.

Montero, G. (2017). Gamification in project management.: Experiences from business and training. En AEIPRO (Ed.), 21th International Congress on Project Management and Engineering. Cádiz: AEIPRO.

Montero, G. (2018). Gamificación en la gestión de proyectos: experiencias desde la formación. En A. Pastor-Fernández y J. L. Yagüe-Blanco (Eds.), Nuevas tendencias de enseñanza aprendizaje aplicadas a la formación en Dirección de Proyectos (pp. 126-162). Cádiz: Universidad de Cádiz y AEIPRO.

Moody, D.L. y Sindre, G. (2003). Evaluating the effectiveness of learning interventions: an information systems case study. ECIS 2003 Proceedings, 80.

Morcillo, P. y Alcachud, M.C. (2005). Creatividad que estás en los cielos... Revista Madrid+D, 30, 1-26.

Neubert, J.C., Mainert, J., Kretzschmar, A. y Greiff, S. (2015). The Assessment of 21st century skills in industrial and organizational psychology: Complex and collaborative problem solving. Industrial and Organizational Psychology: Perspectives on Science and Practice, 8(2), 238-268. https://doi.org/10.1017/iop.2015.14.

Omidvar, G., Samad, Z.A. y Alias, A. (2014). A framework for job-related competencies required for project managers. International Journal of Research in Management y Technology, 4(1), 1-10.

Onisk, M. (2011). Is measuring soft-skills training really possible? Sidney.

Petri, G. y Gresse-von-Wangenheim, C. (2016). How to evaluate educational games: A systematic literature review. Journal of Universal Computer Science, 22(7), 992-1021.

PMI (Project Management Institute). (2017a). Guía de los fundamentos para la dirección de proyectos PMBOK (6 $6^{\mathrm{a}}$ ed.). Newtown Square: PMI.

PMI (Project Management Institute). (2017b). Project manager competency development framework ( $3^{\mathrm{a}}$ ed.). Newtown Square: PMI.
Rainsbury, E., Hodges, D., Burchell, N. y Lay, M. (2002). Ranking workplace competencies: Student and graduate perceptions. Asia-Pacific Journal of Cooperative Education, 3(2), 8-18.

Rasmussen, R. (2010). When you build in the world, you build in your mind. Design Management Review, 17(3), 56-63. https://doi.org/10.1111/j.1948-7169.2006. tb00053.x

Rodríguez, G., Ibarra, M.S. y Cubero, J. (2018). Competencias básicas relacionadas con la evaluación. Un estudio sobre la percepción de los estudiantes universitarios. Educación XX1, 21(1), 181-208. https://doi.org/10.5944/ educXX1.14457.

Roos, J., Victor, B. y Statler, M. (2004). Playing seriously with strategy. Long Range Planning, 37(6), 549-568. https://doi.org/10.1016/j.lrp.2004.09.005.

Ruiz, L., Gordo, M., Fernández, M., Boza, A., Cuenca, L. y Alemany, M.M. (2015). Implementación de actividades de aprendizaje y evaluación para el desarrollo de competencias genéricas : un caso práctico de aplicación de técnicas de pensamiento de diseño y evaluación mediante rúbricas de las competencias de creatividad e innovación. En Congreso Nacional de Innovación Educativa y de Docencia en Red. In-Red (p. 27). Valencia: Universitat Politècnica de València. https://doi.org/10.4995/ INRED2015.2015.1639.

Schulz, K.P., Geithner, S., Woelfel, C. y Krzywinski, J. (2015). Toolkit-based modelling and serious play as means to foster creativity in innovation processes. Creativity and Innovation Management, 24(2), 323-340. https://doi.org/10.1111/caim.12113.

Serrano, M. y Blázquez, P. (2015). Design thinking: Lidera el presente. Crea el futuro ( $1^{\text {a }}$ ed.). Madrid: ESIC Business y Marketing School.

Shen, X.X., Tan, K.C. y Xie, M. (2000). An integrated approach to innovative product development using Kano's model and QFD. European Journal of Innovation Management, 3(2), 91-99. https://doi. org/10.1108/14601060010298435.

Sweetser, P. y Wyeth, P. (2005). GameFlow: A model for evaluating player enjoyment in games. Computers in Entertainment, 3(3), 3-3. https://doi. org/10.1145/1077246.1077253.

Takey, S. M. y de Carvalho, M.M. (2015). Competency mapping in project management: An action research study in an engineering company. International Journal of Project Management, 33(4), 784-796. https://doi. org/10.1016/j.ijproman.2014.10.013. 
Villamizar, G. y González, J.E. (2015). El Lego Serious Play como herramienta para solucionar problemas sociales. Estudio con alumnos de administración de empresas. Cultura, Educación y Sociedad, 6(1), 9-24. https://doi. org/10.5944/ried.19.2.15624.

Vukomanović, M., Young, M. y Huynink, S. (2016). IPMA ICB 4.0: A global standard for project, programme and portfolio management competences. International Journal of Project Management, 34(8), 1703-1705. https:// doi.org/10.1016/j.ijproman.2016.09.011.
Xu, Q., Jiao, R.J., Yang, X., Helander, M., Khalid, H.M. y Opperud, A. (2009). An analytical Kano model for customer need analysis. Design Studies, 30(1), 87-110. https://doi.org/10.1016/j.destud.2008.07.001.

\section{Anexo 1}

Tabla A1-1 Preguntas del cuestionario

variante del modelo de Kirkpatrick.

\begin{tabular}{|c|c|c|c|c|c|c|}
\hline & & $\begin{array}{c}\text { Totalmente } \\
\text { en desacu- } \\
\text { erdo }\end{array}$ & $\begin{array}{l}\text { En desacu- } \\
\quad \text { erdo }\end{array}$ & $\begin{array}{c}\text { Ni en desac- } \\
\text { uerdo ni de } \\
\text { acuerdo }\end{array}$ & De acuerdo & $\begin{array}{l}\text { Totalmente } \\
\text { de acuerdo }\end{array}$ \\
\hline \multicolumn{7}{|c|}{ Motivación. Valore las siguientes afirmaciones } \\
\hline 1. & El diseño del juego me parece atractivo & O & O & O & O & O \\
\hline 2. & Había algo interesante al principio del juego que llamó mi atención & O & O & O & O & O \\
\hline 3. & $\begin{array}{l}\text { La variación (forma, contenido, actividades) me ayudó a mantener la atención } \\
\text { en el juego }\end{array}$ & O & O & O & O & ○ \\
\hline 4. & El contenido del juego es interesante & O & O & O & O & O \\
\hline 5. & La forma en que funciona el juego se adapta a mi manera de aprender & O & O & O & O & O \\
\hline 6. & El contenido del juego está conectado a otros conocimientos que ya tengo & O & O & O & O & O \\
\hline 7. & Fue fácil entender y comenzar a usarlo como material de estudio & O & O & O & O & O \\
\hline \multirow[t]{2}{*}{8.} & Jugando, me sentía seguro de que estaba aprendiendo & & & & & \\
\hline & & O & O & O & O & O \\
\hline 9. & $\begin{array}{l}\text { Estoy satisfecho porque sé que tendré oportunidades para usar en la práctica las } \\
\text { cosas que aprendí en este juego }\end{array}$ & O & O & O & O & O \\
\hline 10. & He logrado avanzar en el juego gracias a mi esfuerzo & O & O & O & O & O \\
\hline \multicolumn{7}{|c|}{ Experiencia del juego. Valore las siguientes afirmaciones } \\
\hline 11. & He estado completamente concentrado en el juego & O & O & O & O & O \\
\hline 12. & He desconectado de mi alrededor & O & O & O & O & O \\
\hline 13. & No me di cuenta de cómo pasaba el tiempo mientras jugaba & O & O & O & O & O \\
\hline 14. & He podido interactuar con otros & o & O & O & O & o \\
\hline
\end{tabular}




\begin{tabular}{|c|c|c|c|c|c|c|}
\hline 15. & Me divertí con otras personas & O & O & O & O & O \\
\hline 16. & $\begin{array}{l}\text { El juego promueve momentos de cooperación y/o competencia entre los ju- } \\
\text { gadores }\end{array}$ & O & O & O & 0 & 0 \\
\hline 17. & $\begin{array}{l}\text { Es un desafío apropiado para mí; las tareas no son demasiado fáciles, ni dema- } \\
\text { siado difíciles }\end{array}$ & ○ & $\bigcirc$ & $\bigcirc$ & $\bigcirc$ & O \\
\hline \multirow[t]{2}{*}{18.} & El juego avanza a un ritmo adecuado y no monótono & & & & & \\
\hline & & O & O & 0 & O & O \\
\hline 19. & El juego ofrece nuevos retos, situaciones o variaciones en sus tareas & $\bigcirc$ & $\bigcirc$ & $\bigcirc$ & 0 & O \\
\hline 20. & Me divertí con el juego & $\bigcirc$ & $\bigcirc$ & $\bigcirc$ & $\bigcirc$ & 0 \\
\hline 21. & Al terminar el juego quería jugar otra partida más & $\bigcirc$ & $\bigcirc$ & $\bigcirc$ & $\bigcirc$ & 0 \\
\hline 22. & Recomendaría este juego a otras personas & $\bigcirc$ & $\bigcirc$ & $\bigcirc$ & $\bigcirc$ & $\bigcirc$ \\
\hline \multicolumn{7}{|c|}{ Conocimientos. Valore las siguientes afirmaciones } \\
\hline 23. & Logré los objetivos del juego aplicando mis conocimientos & ○ & 0 & O & $\bigcirc$ & $\mathrm{O}$ \\
\hline 24. & Tengo una experiencia positiva sobre la eficacia de este juego & $\bigcirc$ & $\bigcirc$ & $\mathrm{O}$ & $\bigcirc$ & ○ \\
\hline 25. & El juego ha contribuido en mi aprendizaje & ○ & ○ & ○ & 0 & O \\
\hline 26. & $\begin{array}{l}\text { El juego ha sido más eficiente en reforzar mi aprendizaje en comparación con } \\
\text { otras actividades del curso }\end{array}$ & 0 & 0 & 0 & 0 & 0 \\
\hline 27. & $\begin{array}{l}\text { La experiencia con el juego va a contribuir a mi desempeño profesional en la } \\
\text { práctica }\end{array}$ & O & 0 & O & O & 0 \\
\hline 28. & Recuerdo la teoría aprendida después de jugar & 0 & 0 & 0 & 0 & 0 \\
\hline
\end{tabular}

\section{Anexo 2}

Tras la puesta en común después de construir el puente-pasarela ESI/UCA-Las Aletas con material LSP, se invita a los jugadores, usuarios potenciales del mismo, que contesten, individual y anónimamente, una encuesta en 2 partes para valorar, a partir de los consensos alcanzados, los requisitos que debería incorporar el puente-pasarela en la vida real:

- La primera parte se fundamenta en un cuestionario estructurado en 3 bloques, como recoge la Tabla A2-1, acerca de la opinión de incorporar o no una serie de atributos y/o especificaciones, para lo que se les ofrece 5 posibilidades: 1) Me gusta, 2) Es algo básico, 3) Me da igual, 5) No me gusta (pero lo tolero) y 6) No me gusta (y no lo tolero).
- $\quad$ La segunda parte consiste en atribuir el grado de importancia $(\mathrm{G})$ para cada requerimiento listado, en una escala $0-10$, siendo 0 nada importante y 10 extremadamente importante, con el objeto de recopilar, filtrar y seleccionar información precisa para valorar si un requisito puede ser considerado como importante desde el punto de vista de los usuarios. 
Tabla A2-1 Preguntas del cuestionario para la recopilación de requisitos del puente-pasarela.

\section{Requisitos urbanos}

$1 \quad$ ¿Te gustaría que SÍ se integrara con el entorno? ¿ ¿ ¿

¿Te gustaría que NO tenga un ascensor?

Requisitos funcionales

\begin{tabular}{|c|c|c|}
\hline 9 & ¿Te gustaría que SÍ tuviera una exposición? & ¿Te gustaría que NO tuviera una exposición? \\
\hline 10 & ¿Te gustaría que SÍ esté climatizada? & ¿Te gustaría que NO esté climatizada? \\
\hline 11 & ¿Te gustaría que SÍ tenga auto-iluminación? & ¿Te gustaría que NO tenga auto-iluminación? \\
\hline 12 & ¿Te gustaría que Sí sea sostenible en consumo? & ¿Te gustaría que NO sea sostenible en consumo? \\
\hline 13 & ¿Te gustaría que SÍ permita el paso de bicicletas? & ¿Te gustaría que NO permita el paso de bicicletas? \\
\hline 14 & ¿Te gustaría que SÍ tenga cobertura WiFi? & ¿Te gustaría que NO tenga cobertura WiFi? \\
\hline
\end{tabular}

El cuestionario se realiza siguiendo las directrices establecidas del método Kano (Kano et al., 1984), orientado a la evaluación de los requisitos de diseño de un producto, teniendo en cuenta 2 dimensiones, permitiendo identificar las características de cada producto. Por un lado, considera el grado de rendimiento del producto y, por otro lado, el grado de satisfacción del cliente, lo que posibilita una evaluación de requisitos clasificándolos en 4 grupos: obligatorios, atractivos, unidimensionales e indiferentes (Matzler y Hinterhuber, 1998). En este contexto, un requisito se considera obligatorio si su ausencia provoca insatisfacción, aunque su presencia se dé por sentada y, por tanto, su inclusión no se valore especialmente. Asimismo, un requisito se denomina atractivo si los clientes y/o usuarios lo valoran cuando está presente, aunque no noten su ausencia y unidimensional si, finalmente, aumenta la satisfacción del cliente de modo proporcional al incremento de su funcionalidad. Por el contrario, si los clientes y/o usuarios no están interesados en un requisito, por secundario, neutral y/o de bajo impacto, entonces este se clasifica como indiferente (Shen et al., 2000).
A continuación, se muestran los resultados del cuestionario, analizando las respuestas proporcionadas por el alumnado de $4^{\circ}$ curso de grado, tanto de la Facultad de Ciencias del Mar y Ambientales (CASEM) como de la Escuela Superior de Ingeniería (ESI), potenciales usuarios del nuevo puente peatonal, respecto a requisitos urbanos, constructivos y funcionales. Para ello, se realizan un total de 9 sesiones, una para cada una de las titulaciones encuestadas, tanto de Ciencias (Ambientales y del Mar) como de Ingeniería (Aeroespacial, Diseño Industrial, Eléctrica, Electrónica Industrial, Mecánica, Química y Tecnología Industrial), con un total de 380 participantes.

Antes de empezar a desarrollar la herramienta, es necesario comprobar la adecuación del tamaño muestral obtenido, que puede determinarse a través de la fórmula (1) para datos globales indicada (Lenth, 2001), en la que para un nivel de confianza del 95\%, y con una población universo $(\mathrm{N})$ de unos 10.000 potenciales usuarios, entre estudiantes, docentes e investigadores (PDI) y personal de administración y servi- 
cios (PAS), con una heterogeneidad máxima del 50\% (por lo que tanto p como q se toman al 50\%) y con un margen de error (e) límite del 5\%; resulta, consecuencia de todo ello, un tamaño mínimo de 370 personas, por lo que las 9 sesiones se consideran suficientes, cubriendo las disparidades posibles.

$$
\frac{K^{2} \times N \times p \times q}{\left[e^{2} \times(N-1)+k^{2} \times p \times q\right]}
$$

Una vez comprobado el tamaño de la muestra, es necesario codificar las opiniones de los participantes (como usuarios potenciales), en función de la respuesta dada para requisito, tanto en positivo como en negativo, según las posibilidades de respuesta antes enumeradas y de acuerdo con la Tabla A22 .

$\begin{array}{ll}\text { Tabla A2-2 Codificación de respuestas } \\ \text { según modelo Kano. } \\ \text { Siendo: } \\ V: & \text { Pregunta inversa } \\ D: & \text { Respuesta dudosa } \\ \text { A: } & \text { Requisitos atractivos } \\ \text { O: } & \text { Requisitos obligatorios } \\ U: & \text { Requisitos unidimensionales } \\ I: & \text { Requisitos indiferentes }\end{array}$

Requisitos funcionales (preguntas positivas)

\begin{tabular}{|c|c|c|c|c|c|c|}
\hline & & \multicolumn{5}{|c|}{$\begin{array}{l}\text { Requisitos disfuncionales } \\
\text { (preguntas negativas) }\end{array}$} \\
\hline & & 1 & 2 & 3 & 4 & 5 \\
\hline \multirow{5}{*}{$\begin{array}{l}\text { Requisitos funcionales } \\
\text { (preguntas positivas) }\end{array}$} & 1 & D & A & A & A & $\mathrm{U}$ \\
\hline & 2 & $\mathrm{~V}$ & I & I & I & $\mathrm{O}$ \\
\hline & 3 & $\mathrm{~V}$ & I & I & I & $\mathrm{O}$ \\
\hline & 4 & V & I & I & I & $\mathrm{O}$ \\
\hline & 5 & $\mathrm{~V}$ & $\mathrm{~V}$ & $\mathrm{~V}$ & $\mathrm{~V}$ & D \\
\hline
\end{tabular}

A continuación, en base al total de respuestas ( $\mathrm{T}$ ) ya codificadas, se realiza un estudio básico de clasificación de requisitos, sintetizado en la Tabla A2-3, a partir de su frecuencia. No obstante, debido a que el cuestionario Kano se utiliza en un contexto donde tiene primacía un enfoque eminentemente cualitativo, se cuenta con una prueba estadística que evalúe lo significativo de la clasificación realizada. Para ello, se utiliza el estadístico Q, siguiendo las indicaciones de la fórmula (2), comparando la diferencia absoluta (F) de las dos frecuencias (a y b) más votadas de las alternativas $(\mathrm{V}, \mathrm{D}, \mathrm{A}, \mathrm{O}$, $\mathrm{U}$, e I) con dicho valor y comprobando que esta es mayor que este, existiendo una diferencia significativa (Fong, 1996).

$Q=\sqrt{\frac{(a+b) \times(2 N-a-b)}{2 N}}$
A partir del cumplimiento de este evaluador estadístico, se procede a la clasificación de los requisitos basada en el aumento o disminución de la satisfacción del usuario debido a la inclusión o no del requisito en el producto final, para lo que se utilizan otros 2 estadísticos, de acuerdo a las fórmulas (3-4). Por un lado, la percepción de ser mejor con su inclusión que sin ella (M) al satisfacer requisitos atractivos y unidimensionales, y, por otro lado, la percepción de ser peor sin su inclusión que con ella, no satisfaciendo requisitos obligatorios ni unidimensionales (Berger et al., 1993). En ambas ecuaciones no se incluyen las respuestas de pregunta inversa y respuesta dudosa, precisamente por su carácter confuso.

$$
\begin{aligned}
& M=\frac{(A+U)}{(A+U+O+1)} \\
& P=\left|\frac{-(U+O)}{(A+U+O+1)}\right|
\end{aligned}
$$


Tabla A2-3 Clasificación funcional/ disfuncional de requisitos.

Siendo:

T: $\quad$ Respuestas totales.

Q: $\quad$ Estadístico de comprobación.

M: $\quad$ Mejor (incremento de satisfacción).

F: $\quad$ Diferencia frecuencias más votadas.

P: $\quad$ Peor (decremento de satisfacción).

C: $\quad$ Clasificación preliminar

No obstante, algunos aspectos sobre el análisis de necesidades de los usuarios no están completamente resueltos:

- Evaluación cuantitativa de la satisfacción del usuario.

- $\quad$ Soporte de decisiones de diseño y ejecución.

- Incorporación de la evaluación de la capacidad de las contratas.

Con el propósito de mejorar estos aspectos, el modelo analítico A-Kano (Xu et al., 2009) sigue los principios básicos del modelo tradicional, pero tratando de consolidar la fundamentación teórica. En primer lugar, se puntúan las preguntas funcionales y disfuncionales, como muestra la Tabla A2-4. En segundo lugar, se calcula el vector (X, Y) para cada requisito (i), de acuerdo a las fórmulas (5-6) y las puntuaciones referidas. En tercer lugar, se corrige el vector $X Y$ ponderándolo con el grado de importancia (G) normalizado, determinando los valores C1, C2. Estos parámetros, se muestran en una gráfica (), en una escala bidimensional funcional-disfuncional, para clasificar el tipo de requisito, como resume la Tabla A2-5.

$$
\begin{aligned}
& X_{i}=\frac{1}{380} \sum_{j=1}^{380} x_{i j} \\
& Y_{i}=\frac{1}{380} \sum_{j=1}^{380} y_{i j}
\end{aligned}
$$


Guillermo Montero et al. / Dirección y Organización 69 (2019) 36-61

Tabla A2-4 Puntuación de respuestas funcionales y disfuncionales.

Tabla A2-5 Clasificación analítica de requisitos según método A-Kano.

* Estos requisitos se sitúan cerca de la frontera de las regiones adyacentes A-U

\begin{tabular}{lcc}
\multicolumn{1}{c}{ Respuestas } & Pregunta funcional & Pregunta disfuncional \\
\hline Me gusta & 1,00 & $-0,50$ \\
\hline & & \\
Es algo básico & 0,50 & $-0,25$ \\
\hline & & 0,00 \\
\hline Me da igual & 0,00 & 0,50 \\
\hline & & \\
No me gusta, pero lo tolero & $-0,25$ & 1,00 \\
\hline
\end{tabular}

\begin{tabular}{|c|c|c|c|c|c|c|c|}
\hline Código & Denominación & $G_{i}$ & $\mathbf{X}_{\mathrm{i}}$ & $Y_{i}$ & $\mathrm{C1}_{\mathrm{i}}$ & $\mathrm{C}_{\mathrm{i}}$ & $\mathrm{C}_{\mathrm{i}}$ \\
\hline 1 & $\begin{array}{c}\text { Integración en } \\
\text { el entorno }\end{array}$ & 8,98 & 0,68 & 0,47 & 0,61 & 0,42 & $\mathrm{O}$ \\
\hline 2 & $\begin{array}{l}\text { Diseño inno- } \\
\text { vador }\end{array}$ & 9,21 & 0,61 & 0,32 & 0,56 & 0,30 & $\mathrm{O}$ \\
\hline 3 & Cubrición & 9,13 & 0,70 & 0,40 & 0,64 & 0,37 & $\mathrm{O}$ \\
\hline 4 & Insonorización & 6,57 & 0,26 & 0,17 & 0,17 & 0,11 & I \\
\hline 5 & Anti-vibración & 5,74 & 0,63 & 0,64 & 0,36 & 0,37 & $I^{*}$ \\
\hline 6 & Antivandalismo & 6,26 & 0,67 & 0,58 & 0,42 & 0,36 & $I^{*}$ \\
\hline 7 & $\begin{array}{c}\text { Control de } \\
\text { accesos }\end{array}$ & 6,85 & 0,04 & 0,07 & 0,03 & 0,05 & I \\
\hline 8 & Ascensor & 8,70 & 0,49 & 0,60 & 0,43 & 0,52 & A \\
\hline 9 & $\begin{array}{c}\text { Exposiciones } \\
\text { itinerantes }\end{array}$ & 7,22 & 0,27 & 0,09 & 0,19 & 0,07 & I \\
\hline 10 & Climatización & 5,27 & 0,09 & 0,01 & 0,05 & 0,00 & I \\
\hline 11 & $\begin{array}{l}\text { Iluminación } \\
\text { automatizada }\end{array}$ & 8,36 & 0,75 & 0,62 & 0,62 & 0,52 & $\mathrm{U}$ \\
\hline 12 & $\begin{array}{l}\text { Consumo } \\
\text { sostenible }\end{array}$ & 9,07 & 0,74 & 0,59 & 0,67 & 0,54 & $\mathrm{U}$ \\
\hline 13 & Carril bici & 8,71 & 0,74 & 0,73 & 0,64 & 0,63 & $\mathrm{U}$ \\
\hline 14 & $\begin{array}{c}\text { Cobertura WiFi } \\
\text { UCA }\end{array}$ & 7,96 & 0,36 & 0,14 & 0,29 & 0,11 & I \\
\hline
\end{tabular}


Figura A2-1 Clasificación funcional/ disfuncional de requisitos.

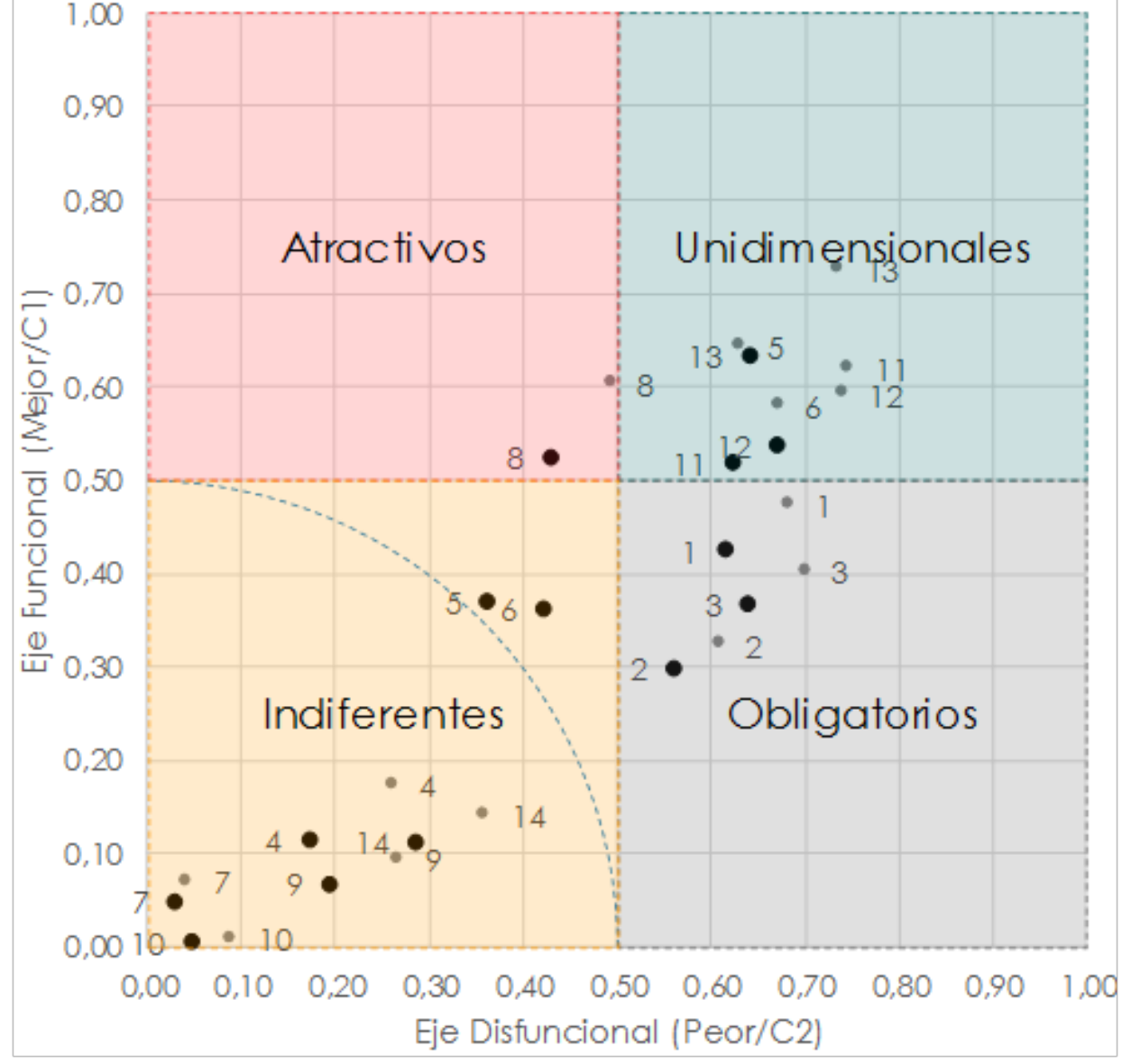

A partir de la Figura A2-1, se clasifican los requisitos analíticamente vía método A-Kano, entre indiferentes (4, 5*, 6*, 7, 9,14), atractivos (8), unidimensionales $(11,12,13)$ у obligatorios (1, 2, 3). Es relevante precisar que, como indica la Tabla A2-6, si sola se hubiera preguntado la importancia relativa una serie de atributos, la información proporcionada hubiera conducido a considerar como necesarios algunos requisitos resultantes como opcionales, y como mandatorios otros que, contextualizados, acaban provocando indiferencia. 
Tabla A2-6 Clasificación analítica de requisitos según método A-Kano.

Siendo la evolución $\rightarrow$ :

$=\quad$ Requisitos que no modifican su condición tras el estudio Requisitos que símodifican su condición tras el estudio (siendo no críticos)

$<\quad$ Requisitos que símodifican su condición tras el estudio (notoriamente)

\begin{tabular}{|c|c|c|c|c|c|c|c|c|c|c|c|}
\hline Código & $\begin{array}{c}\text { Denomi- } \\
\text { nación }\end{array}$ & G & Kano & $\rightarrow$ & A-Kano & Código & $\begin{array}{c}\text { Denomi- } \\
\text { nación }\end{array}$ & G & Kano & $\rightarrow$ & A-Kano \\
\hline 1 & Integración & MP & $\mathrm{O}$ & $=$ & $\mathrm{O}$ & 8 & Ascensor & MP & $\mathrm{U}$ & $<$ & A \\
\hline 2 & $\begin{array}{c}\text { Diseño } \\
\text { innovador }\end{array}$ & EP & $\mathrm{O}$ & $=$ & $\mathrm{O}$ & 9 & $\begin{array}{l}\text { Exposi- } \\
\text { ciones }\end{array}$ & MP & I & $=$ & I \\
\hline 3 & Cubrición & EP & $\mathrm{O}$ & $=$ & $\mathrm{O}$ & 10 & $\begin{array}{l}\text { Clima- } \\
\text { tización }\end{array}$ & I & I & $=$ & I \\
\hline 4 & $\begin{array}{c}\text { Inso- } \\
\text { norización }\end{array}$ & I & I & $=$ & I & 11 & $\begin{array}{l}\text { Auto-ilu- } \\
\text { minación }\end{array}$ & MP & $\mathrm{O}$ & $<$ & $\mathrm{U}$ \\
\hline 5 & $\begin{array}{l}\text { Anti-vi- } \\
\text { bración }\end{array}$ & I & A & $<$ & $\mathrm{I}^{*}$ & 12 & $\begin{array}{l}\text { Sostenibi- } \\
\text { lidad }\end{array}$ & $\mathrm{EP}$ & $\mathrm{O}$ & $<$ & $\mathrm{U}$ \\
\hline 6 & $\begin{array}{l}\text { Antivandal- } \\
\text { ismo }\end{array}$ & I & $\mathrm{O}$ & $<<$ & $\mathrm{I}^{*}$ & 13 & Carril bici & MP & $\mathrm{U}$ & $=$ & $\mathrm{U}$ \\
\hline 7 & $\begin{array}{l}\text { Control de } \\
\text { accesos }\end{array}$ & I & I & $=$ & I & 14 & $\begin{array}{c}\text { Cobertura } \\
\text { WiFi }\end{array}$ & MP & $\mathrm{O}$ & $<<$ & I \\
\hline
\end{tabular}

